Annals of Mathematics, 156 (2002), 797-833

\title{
An infinite Ramsey theorem and some Banach-space dichotomies
}

\author{
By W. T. GOwers
}

\begin{abstract}
A problem of Banach asks whether every infinite-dimensional Banach space which is isomorphic to all its infinite-dimensional subspaces must be isomorphic to a separable Hilbert space. In this paper we prove a result of a Ramsey-theoretic nature which implies an interesting dichotomy for subspaces of Banach spaces. Combined with a result of Komorowski and TomczakJaegermann, this gives a positive answer to Banach's problem. We then generalize the Ramsey-theoretic result and deduce a further dichotomy for Banach spaces with an unconditional basis.
\end{abstract}

\section{Introduction}

This paper contains a complete proof of a result announced in [G1] which has been circulating in preprint form for several years [G2,3]. Our main theorem, when combined with a very different result of Komorowski and TomczakJaegermann, solves a problem from the famous 1932 book of Banach [B]. He asked whether a separable Hilbert space is the only infinite-dimensional Banach space, up to isomorphism, which is isomorphic to every infinite-dimensional closed subspace of itself. The answer turns out to be yes, but most of the proof appears to have little to do with the problem. Therefore, in order to motivate the rest of the paper, we shall begin by explaining (in this section and the next) how Banach's problem can be reduced to a question with a much more combinatorial flavour.

To save writing, let us assume from now on that all Banach spaces and subspaces are infinite-dimensional unless they are specified otherwise. Recall that a (Schauder) basis of a Banach space $X$ is a sequence $\left(x_{n}\right)_{n=1}^{\infty}$ such that every $a \in X$ can be written uniquely as a norm-convergent sum $a=\sum_{n=1}^{\infty} a_{n} x_{n}$ for some sequence $\left(a_{n}\right)_{n=1}^{\infty}$ of scalars. If every $x_{n}$ has norm one then the basis is normalized. It can be shown that if $\left(x_{n}\right)_{n=1}^{\infty}$ is a basis, then there is a constant $C$ such that all the projections $P_{N}: \sum_{n=1}^{\infty} a_{n} x_{n} \mapsto \sum_{n=1}^{N} a_{n} x_{n}$ have norm at 
most $C$. The smallest constant with this property is called the basis constant of the basis. If the basis constant is 1 , then the basis is called monotone. A basis $\left(x_{n}\right)_{n=1}^{\infty}$ is unconditional if the sum $\sum_{n=1}^{\infty} a_{n} x_{n}$ converges unconditionally whenever it converges. This, it turns out, implies that there is a constant $C$ such that, for every subset $A \subset \mathbb{N}$ (it is sufficient to consider only finite subsets), the projection $P_{A}: \sum_{n=1}^{\infty} a_{n} x_{n} \mapsto \sum_{n \in A} a_{n} x_{n}$ has norm at most $C$. The smallest constant with this property is called the unconditional constant of the basis. A sequence $\left(x_{n}\right)_{n=1}^{\infty}$ which is a basis/unconditional basis of the closed subspace that it spans is called a basic sequence/unconditional basic sequence.

The result of Komorowski and Tomczak-Jaegermann we shall use is the following [K T-J].

THEOREM 1.1. Let $X$ be a Banach space with cotype $q$ for some $q<\infty$. Then either $X$ has a subspace without an unconditional basis or $X$ has a subspace isomorphic to $\ell_{2}$.

Let us follow usual practice and define a Banach space to be homogeneous if it is isomorphic to all its subspaces. It can be shown that every homogeneous Banach space satisfies the cotype condition above. Indeed, an old result of Mazur states that every Banach space has a subspace with a basis. On the other hand, Szankowski has shown [Sz] (generalizing Enflo's solution of the basis problem) that if $p<2$ and $q>2$, then any Banach space which either fails to have type $p$ or fails to have cotype $q$ has a subspace without a basis. Hence, such a space cannot be homogeneous. So an immediate consequence of Theorem 1 and the definition of homogeneity is the following additional result of Komorowski and Tomczak-Jaegermann.

Corollary 1.2. Let $X$ be a homogeneous Banach space. Then either $X$ is isomorphic to $\ell_{2}$ or $X$ fails to have an unconditional basis.

Notice that in the second case no subspace of $X$ has an unconditional basis, since $X$ is homogeneous. Thus Corollary 1.2 appears to imply a very strong property of homogeneous spaces not isomorphic to $\ell_{2}$. Indeed, it is not at all obvious that there exists a Banach space such that no subspace has an unconditional basis.

As it happens, the existence of such spaces was itself a long-standing open problem until 1991, when counterexamples were found [GM1] (see also [AD], [F1], [G6], [GM2], [H]). However, these counterexamples did not come close to being homogeneous. In fact, many of them had a property which is almost the reverse of homogeneity. A Banach space $X$ is called decomposable if it can be written as a direct sum $Y+Z$ with the projections to $Y$ and $Z$ continuous. Equivalently, $X$ is decomposable if it admits a nontrivial projection (that is, 
one of infinite rank and infinite corank). Otherwise, $X$ is indecomposable. It is hereditarily indecomposable if it has no decomposable subspace. This is the remarkable property which was enjoyed by many of the counterexamples. Later we shall have more to say about hereditarily indecomposable spaces, but for now we shall simply quote the following result from [GM1].

TheOREM 1.3. A hereditarily indecomposable Banach space is isomorphic to no proper subspace of itself.

In particular, a hereditarily indecomposable space is certainly not homogeneous.

We can now state our first Banach-space dichotomy, which is one of the main results of this paper.

THEOREM 1.4. Every Banach space $X$ has a subspace $W$ which either has an unconditional basis or is hereditarily indecomposable.

Note that this is a genuine dichotomy, since an unconditional basis allows $W$ to be decomposed in uncountably many different ways. Note also that the last two results imply that a homogeneous space $X$ must have an unconditional basis, since otherwise no subspace of $X$ has an unconditional basis, which implies, by Theorem 1.4, that some subspace of $X$ is hereditarily indecomposable, which implies, by homogeneity, that $X$ is hereditarily indecomposable, which implies, by Theorem 1.3, that $X$ is not homogeneous. However, we have already seen that a homogeneous space with an unconditional basis must be isomorphic to $\ell_{2}$, so Banach's problem is solved once Theorem 1.4 is established.

The definitions we have given of an unconditional basis and a hereditarily indecomposable Banach space are not the most convenient for proving Theorem 1.4. Instead, we use simple characterizations of these concepts in terms of finite sequences of vectors in the space. First we need some more definitions and elementary facts concerned with bases.

Because of Mazur's result that every Banach space has a subspace with a basis, and indeed a basis with basis constant arbitrarily close to 1 , we shall restrict attention to spaces with a monotone basis. Two bases $\left(x_{n}\right)_{n=1}^{\infty}$ and $\left(y_{n}\right)_{n=1}^{\infty}$ are defined to be $C$-equivalent if there exist constants $A$ and $B$ such that $B / A \leqslant C$ and

$$
A\left\|\sum_{n=1}^{\infty} a_{n} x_{n}\right\| \leqslant\left\|\sum_{n=1}^{\infty} a_{n} y_{n}\right\| \leqslant B\left\|\sum_{n=1}^{\infty} a_{n} x_{n}\right\|
$$

for every sequence of scalars $\left(a_{n}\right)_{n=1}^{\infty}$ such that either of the two sums converges. If $X$ is a Banach space with a given basis $\left(e_{n}\right)_{n=1}^{\infty}$, then the support of a vector $x=\sum_{n=1}^{\infty} x_{n} e_{n}$, written $\operatorname{supp}(x)$, is the set of $n$ for which $x_{n} \neq 0$. 
If $\max \operatorname{supp}(x)<\min \operatorname{supp}(y)$ we write $x<y$. A block basis of $X$ is defined to be a sequence $x_{1}<x_{2}<\ldots$ of nonzero vectors. A subspace of $X$ generated by a block basis is called a block subspace. The following very useful lemma of Bessaga and Pelczynski (see [LT]) allows us to restrict our attention to block bases and block subspaces.

Lemma 1.5. Let $X$ be a Banach space with a basis $\left(e_{n}\right)_{n=1}^{\infty}$, let $Y$ be a subspace of $X$ and let $\varepsilon>0$. Then $Y$ has a subspace $Z$ generated by a basis which is $(1+\varepsilon)$-equivalent to a block basis of $\left(e_{n}\right)_{n=1}^{\infty}$.

In fact, more is true. For every sequence $\left(\delta_{n}\right)_{n=1}^{\infty}$ of positive real numbers, we can find a subspace $Z$ with a basis $\left(z_{n}\right)_{n=1}^{\infty}$ such that there is a normalized block basis $\left(x_{n}\right)_{n=1}^{\infty}$ of $\left(e_{n}\right)_{n=1}^{\infty}$ with $\left\|x_{n}-z_{n}\right\| \leqslant \delta_{n}$ for every $n$. In other words, $Z$ can be chosen to be an arbitrarily small perturbation of a block subspace of $X$. Moreover, given any basic sequence in $Y$, we can choose $Z$ to be spanned by a subsequence.

We now state two simple lemmas. Proofs can be found in [LT] and [GM1]. From now on, when we use block-basis terminology, we shall assume implicitly that the Banach space under discussion comes with some particular chosen normalized monotone basis $\left(e_{n}\right)_{n=1}^{\infty}$. This will save a good deal of writing.

Lemma 1.6. Let $X$ be a Banach space. The following two statements are equivalent:

(i) X has no subspace with an unconditional basis.

(ii) For every block subspace $Y$ of $X$ and every real number $C$ there is a sequence $y_{1}<\ldots<y_{n}$ of vectors in $Y$ such that

$$
\left\|\sum_{i=1}^{n} y_{i}\right\|>C\left\|\sum_{i=1}^{n}(-1)^{i} y_{i}\right\| .
$$

Lemma 1.7. Let $X$ be a Banach space. The following two statements are equivalent:

(i) $X$ is hereditarily indecomposable.

(ii) For every pair of block subspaces $Y, Z$ of $X$ and every real number $C$ there is a sequence $y_{1}<z_{1}<y_{2}<z_{2}<\ldots<y_{n}<z_{n}$ of vectors such that $y_{i} \in Y$ and $z_{i} \in Z$ for every $i$, and such that

$$
\left\|\sum_{i=1}^{n}\left(y_{i}+z_{i}\right)\right\|>C\left\|\sum_{i=1}^{n}\left(y_{i}-z_{i}\right)\right\| .
$$


Notice that if we insist that $Y=Z$ in condition (ii) of Lemma 1.7 then we recover condition (ii) of Lemma 1.6 (with $n$ replaced by $2 n$ ). This makes it clearer what we need to do to prove Theorem 1.4. Let us say that a sequence $y_{1}<\ldots<y_{n}$ is $C$-conditional if

$$
\left\|\sum_{i=1}^{n} y_{i}\right\|>C\left\|\sum_{i=1}^{n}(-1)^{i} y_{i}\right\| .
$$

Then we begin with a space $X$ such that every block subspace contains a $C$-conditional sequence for every $C$, and must find a (block) subspace with the stronger property that for any two block subspaces and any $C$, a $C$-conditional sequence can be found with its odd terms in one of the subspaces and its even terms in the other. As we shall see later in the paper, a far more general result is true, one which does not use in a strong way the definition of $C$-conditional.

\section{Further definitions and some preliminary results}

In this section we shall state the first of our main Ramsey-theoretic results, which implies Theorem 1.4 very easily. It will be proved in the next section. Given a Banach space $X$ (with a specified basis) let us define $\Sigma_{f}=\Sigma_{f}(X)$ to be the set of all finite sequences $x_{1}<\ldots<x_{n}$ of nonzero vectors in the unit ball of $X$. Given an arbitrary subset $\sigma \subset \Sigma_{f}$ (in other words, given some set of finite block bases) the following two-player game can be defined, between players $\mathrm{S}$ and $\mathrm{P}$. On the $n^{\text {th }}$ move of the game, player $\mathrm{S}$ chooses a block subspace $X_{n} \subset X$ (infinite-dimensional), and player $\mathrm{P}$ chooses some point $x_{n} \in X_{n}$. The aim of $\mathrm{P}$ is to construct a sequence $\left(x_{1}, \ldots, x_{n}\right) \in \sigma$, whereas the aim of $\mathrm{S}$ is that at no stage should the sequence constructed by $\mathrm{P}$ belong to $\sigma$.

Formally, a strategy for $\mathrm{P}$ is a function $\phi$ which, for any finite block basis $x_{1}, \ldots, x_{n}$ and any subspace $Y \subset X$, gives a vector $x=\phi\left(x_{1}, \ldots, x_{n} ; Y\right) \in Y$. The strategy $\phi$ is a winning strategy for $\mathrm{P}$ if, given any sequence $X_{1}, X_{2}, \ldots$ of subspaces of $X$, the sequence $\left(x_{1}, x_{2}, \ldots\right)$ defined inductively by $x_{1}=\phi\left(\emptyset ; X_{1}\right)$ and $x_{n+1}=\phi\left(x_{1}, \ldots, x_{n} ; X_{n+1}\right)$ is in $\sigma$.

If $\Delta=\left(\delta_{1}, \delta_{2}, \ldots\right)$ is a sequence of positive scalars and $\sigma \subset \Sigma_{f}$, then the $\Delta$-expansion of $\sigma$, denoted $\sigma_{\Delta}$, is the set of block bases $\left(x_{1}, \ldots, x_{n}\right) \in \Sigma_{f}$ such that there exists $\left(y_{1}, \ldots, y_{n}\right) \in \sigma$ with $\left\|y_{i}-x_{i}\right\| \leqslant \delta_{i}$ for every $i$. We shall also define $\sigma_{-\Delta}$ to be $\left(\left((\sigma)^{c}\right)_{\Delta}\right)^{c}$, or in other words the set of block bases $\left(x_{1}, \ldots, x_{n}\right) \in \Sigma_{f}$ such that every $\left(y_{1}, \ldots, y_{n}\right) \in \sigma$ with $\left\|y_{i}-x_{i}\right\| \leqslant \delta_{i}$ for all $i$ is in $\sigma$. It is easy to check that $\left(\sigma_{-\Delta}\right)_{\Delta} \subset \sigma \subset\left(\sigma_{\Delta}\right)_{-\Delta}$.

We now introduce some notation. If $A=\left(y_{1}, \ldots, y_{m}\right)$ and $Y \subset X$ is a subspace, then $[A ; Y]$ will stand for the set of sequences $\left(z_{n}\right)_{n=1}^{N} \in \Sigma_{f}$ such that $z_{i}=y_{i}$ for $i \leqslant m$ and $z_{i} \in Y$ for $m<i \leqslant N$. If $A$ is the null sequence then we shall write $[Y]$. Given $\sigma \subset \Sigma_{f}$, we write $\sigma[A ; Y]$ for the set of sequences 
$\left(x_{n}\right)_{n=1}^{N}$ such that $\left(y_{1}, \ldots, y_{m}, x_{1}, \ldots, x_{N}\right) \in[A ; Y] \cap \sigma$. Finally, when we say that $\mathrm{P}$ has a winning strategy for the game $\sigma[A ; Y]$, S's moves are understood to have to be subspaces of $Y$.

Our first Ramsey-type theorem is the following.

Theorem 2.1. Let $X$ be a Banach space, let $\sigma$ be any subset of $\Sigma_{f}(X)$ and let $\Delta$ be a sequence of positive real numbers. Then $X$ has a subspace $Y$ such that either $\sigma[Y]=\emptyset$ or $P$ has a winning strategy for the game $\sigma_{\Delta}[Y]$.

We refer to this as a Ramsey theorem for two reasons. The first is that its proof closely resembles existing arguments in Ramsey theory. However, even the statement can be regarded as Ramsey-theoretical. If we call sequences in $\sigma$ blue and those not in $\sigma$ red, then the theorem gives us a subspace such that either every finite block sequence is red or there is such an abundance of small perturbations of blue sequences that $\mathrm{P}$ has a winning strategy for obtaining them. One might hope to find a subspace where all sequences were close to blue sequences, but a strengthened statement along these lines is false (for nontrivial reasons, see the appendix).

Many arguments in infinite Ramsey theory depend on one particular diagonalization procedure. It will be used often enough in this paper for it to be well worth stating as an abstract principle. We shall need some more notation. A *-pair is a pair $(A, Z)$ where $A \in \Sigma_{f}, Z$ is an infinite-dimensional block subspace and $x<z$ for every $x \in A$ and $z \in Z$. We shall sometimes refer to *-pairs simply as pairs, and we shall use the notation $A<Z$ as shorthand for the support condition above. If $\Delta$ is a sequence $\left(\delta_{1}, \delta_{2}, \ldots\right)$ of positive reals and $A=\left(x_{1}, \ldots, x_{n}\right)$ and $B=\left(y_{1}, \ldots, y_{n}\right)$ are block bases of the same size, we use the shorthand notation $d(A, B) \leqslant \Delta$ to mean $d\left(x_{i}, y_{i}\right) \leqslant \delta_{i}$ for each $i$. The definition of a $\Delta$-net of a set of block bases is obvious. If $\Pi$ is a set of $*$-pairs, we write $\Pi_{\Delta}$ for the set of pairs $(A, Z)$ such that there is a block basis $B$ with $d(A, B) \leqslant \Delta$ and $(B, Z) \in \Pi$. If $x_{1}<\ldots<x_{n}$, then we shall write $\left\langle x_{1}, \ldots, x_{n}\right\rangle$ for the subspace generated by $x_{1}, \ldots, x_{n}$. Given a sequence $\left(x_{1}, \ldots, x_{n}\right) \in \Sigma$, we shall write $\Sigma_{f}\left(x_{1}, \ldots, x_{n}\right)$ for the set of all sequences $\left(y_{1}, \ldots, y_{k}\right) \in \Sigma_{f}$ such that every $y_{i}$ belongs to the subspace $\left\langle x_{1}, \ldots, x_{n}\right\rangle$.

LEMma 2.2. Let $\Delta_{1}, \Delta_{2}, \ldots$ be a sequence of positive real sequences and let $\Pi_{1}, \Pi_{2}$, . . be a sequence of sets of $*$-pairs satisfying the following conditions:

(i) for every pair $(A, Z)$ and every $n$ there is $Z^{\prime} \subset Z$ such that $\left(A, Z^{\prime}\right) \in \Pi_{n}$;

(ii) if $(A, Z) \in \Pi_{n}$ and $Z^{\prime} \subset Z$ then $\left(A, Z^{\prime}\right) \in \Pi_{n}$.

Then there exists a subspace $Y \subset X$ such that $(A, Z) \in\left(\Pi_{n}\right)_{\Delta_{n}}$ for every pair $(A, Z)$ such that $A$ is of length at least $n$ and both $A$ and $Z$ are subsets of $Y$. 
Proof. Choose a block basis $y_{1}, y_{2}, \ldots$ and a sequence of block subspaces $X=Y_{0} \supset Y_{1} \supset Y_{2} \supset \ldots$ inductively as follows. Once we have chosen $y_{1}, \ldots, y_{n-1}$ and $Y_{1}, \ldots, Y_{n-1}$, let $y_{n} \in Y_{n-1}$ be arbitrary (except that $\left.\left(y_{1}, \ldots, y_{n}\right) \in \Sigma_{f}\right)$ and let $A_{1}, \ldots, A_{N}$ be a $\Delta_{n}$-net of the set $\Sigma_{f}\left(y_{1}, \ldots, y_{n}\right)$.

By property (i) we can pick a sequence of subspaces

$$
Y_{n-1} \supset V_{11} \supset \ldots \supset V_{1 n} \supset V_{21} \supset \ldots \supset V_{2 n} \supset \ldots \supset V_{N 1} \supset \ldots \supset V_{N n}
$$

such that $\left(A_{i}, V_{i j}\right) \in \Pi_{j}$ for every $1 \leqslant i \leqslant N$ and $1 \leqslant j \leqslant n$. Let $Y_{n}=V_{N n}$. By property (ii) we have $\left(A_{i}, Z\right) \in \Pi_{j}$ for every $i, j$ and every $Z \subset Y_{n}$. Since $\left(A_{n}\right)_{n=1}^{N}$ is a $\Delta_{n}$-net, we find that $(A, Z) \in\left(\Pi_{j}\right)_{\Delta_{n}}$ for every $j \leqslant n$ and every pair $(A, Z)$ such that $A \in \Sigma\left(y_{1}, \ldots, y_{n}\right)$ and $Z \subset Y_{n}$.

Let $Y=\left\langle y_{1}, y_{2}, \ldots\right\rangle$ and suppose that $(A, Z)$ is a $*$-pair inside $Y$, with $A$ of length $m \geqslant n$. Then there exists $k \geqslant n$ such that $A \subset\left\langle y_{1}, \ldots, y_{k}\right\rangle$ and $Z \subset\left\langle y_{k+1}, y_{k+2}, \ldots\right\rangle \subset Y_{k}$. By our construction therefore, $(A, Z) \in\left(\Pi_{n}\right)_{\Delta_{n}}$. Hence, $Y$ will do.

We shall often use specializations of Lemma 2.2. It may be convenient for the reader if we state them separately. Let us define a singleton $*$-pair to be a pair $(x, Z)$ where $x$ is a nonzero vector of norm at most $1, Z$ is a block subspace and $x<z$ for every $z \in Z$ (or in other words a $*$-pair $(A, Z$ ) for which $A$ is a singleton). Given $\delta>0$ and a set $\Pi$ of singleton $*$-pairs, write $\Pi_{\delta}$ for the set of $*$-pairs $(x, Z)$ for which there exists $x^{\prime}$ such that $d\left(x, x^{\prime}\right) \leqslant \delta$, $x^{\prime}<z$ for every $z \in Z$ and $\left(x^{\prime}, Z\right) \in \Pi$.

COROLlary 2.3. Let $\delta>0$ and let $\Pi$ be a set of singleton $*$-pairs satisfying the following conditions:

(i) for every pair $(y, Z)$ there is $Z^{\prime} \subset Z$ such that $\left(y, Z^{\prime}\right) \in \Pi$;

(ii) if $(y, Z) \in \Pi$ and $Z^{\prime} \subset Z$ then $\left(y, Z^{\prime}\right) \in \Pi$.

Then there exists a subspace $Y \subset X$ such that every pair $(y, Z)$ with $y \in Y$ and $Z \subset Y$ belongs to $\Pi_{\delta}$.

Proof. Apply Lemma 2.2 with the following choices for the $\Pi_{i}$ and $\Delta_{i}$. Let $\Pi_{1}$ be the set of all $*$-pairs $(A, Z)$ such that if $A$ is a singleton, then $(A, Z) \in \Pi$. (Thus, every pair $(A, Z)$ for which $A$ is not a singleton belongs to $\Pi_{1}$.) If $i>1$, then let $\Pi_{i}$ be the set of all $*$-pairs. Let all $\Delta_{i}$ be the sequence $(\delta, 1,1,1, \ldots)$ (but all that matters is that the first term of $\Delta_{1}$ should be $\delta$ ).

Corollary 2.4. Let $\Delta_{1}, \Delta_{2}, \ldots$ and $\Pi_{1}, \Pi_{2}, \ldots$ be as in Lemma 2.2 but satisfying the following additional condition:

(iii) if $(A, Z) \in \Pi_{n}$ then $A$ has length $n$. 
Then there exists a subspace $Y \subset X$ such that $(A, Z) \in\left(\Pi_{n}\right)_{\Delta_{n}}$ for every pair $(A, Z)$ such that $A$ and $Z$ are subsets of $Y$ and $A$ has length $n$.

Proof. Apply Lemma 2.2 replacing each $\Pi_{n}$ by $\Pi_{n}^{\prime}$, where $\Pi_{n}^{\prime}$ is the set of *pairs $(A, Z)$ such that if $A$ has length $n$ then $(A, Z) \in \Pi_{n}$. (As in Corollary 2.3, if $A$ has any other length then $(A, Z)$ belongs to $\Pi_{n}^{\prime}$.)

\section{A Banach-space dichotomy}

In this section we shall prove Theorem 2.1 and show in detail how it implies Theorem 1.4. This will complete the solution of Banach's problem on homogeneous spaces. Before stating the next result, let us make two more definitions. If $X$ is a Banach space, $Y$ is a block subspace of $X$ and $\sigma \subset \Sigma_{f}(X)$, we shall say that $\sigma$ is large for $Y$ if every block subspace of $Y$ contains a sequence in $\sigma$, and strategically large for $Y$ if $\mathrm{P}$ has a winning strategy for the game $\sigma[Y]$. More generally, if $(A, Y)$ is a $*$-pair, we say that $\sigma$ is large for $[A ; Y]$ if every block subspace of $Y$ contains a sequence $B \in \Sigma_{f}$ such that $(A, B) \in \sigma$, and strategically large for $[A ; Y]$ if $\mathrm{P}$ has a winning strategy for the game $\sigma[A ; Y]$. Note that $\sigma$ is (strategically) large for $[A ; Y]$ if and only if $\sigma[A ; Y]$ is (strategically) large for $Y$.

Theorem 3.1. Let $X$ be a Banach space with a given monotone basis. Let $\Theta=\left(\theta_{n}\right)_{n=1}^{\infty}$ and $\Delta=\left(\delta_{n}\right)_{n=1}^{\infty}$ be sequences of positive real numbers such that $2 \sum_{i=N}^{\infty} \delta_{i} \leqslant \theta_{N}$ for every $N$. If $\sigma_{-\Theta}$ is large for $X$, then $X$ has a block subspace $Y$ such that $\sigma_{2 \Delta}$ is strategically large for $Y$.

Proof. Suppose that $\sigma \subset \Sigma_{f}$ is a set for which the result is false. Then $\sigma_{-\Theta}$ is large for $X$, so in particular $\sigma$ is large for $X$; on the other hand, $\sigma_{2 \Delta}$ is not strategically large for any subspace of $X$. Let $\rho$ be the set of sequences $\left(x_{1}, \ldots, x_{n}\right) \in \sigma$ such that if $y_{1}<\ldots<y_{k}$ and $\left\langle y_{1}, \ldots, y_{k}\right\rangle$ is a proper subspace of $\left\langle x_{1}, \ldots, x_{n}\right\rangle$, then $\left(y_{1}, \ldots, y_{k}\right) \notin \sigma$. It is easy to see that $\rho$ is still large for $X$ and that $\rho_{2 \Delta}$ is not strategically large for any subspace of $X$.

For each $n \geqslant 0$ Let $\Delta_{n}=\left(\delta_{1}, \ldots, \delta_{n}, 0,0, \ldots\right)$ and let $\Gamma_{n}=2 \Delta-\Delta_{n}=$ $\left(\delta_{1}, \ldots, \delta_{n}, 2 \delta_{n+1}, 2 \delta_{n+2}, \ldots\right)$. We now construct sequences $x_{1}, x_{2}, \ldots$ and $X=$ $X_{0} \supset X_{1} \supset X_{2} \supset \ldots$ with the following properties for every $n$ :

(i) $x_{n} \in X_{n-1}$;

(ii) $\rho_{\Delta_{n}}$ is large for $\left[x_{1}, \ldots, x_{n} ; X_{n}\right]$;

(iii) $\rho_{\Gamma_{n}}$ is not strategically large for any $\left[x_{1}, \ldots, x_{n} ; Z\right]$ with $Z \subset X_{n}$.

The induction starts with the space $X_{0}$, since $\rho_{\Delta_{0}}$ is large for $X_{0}$ but $\rho_{\Gamma_{0}}$ is not large for any subspace of $X_{0}$. Having found $x_{1}, \ldots, x_{n}$ and $X_{1}, \ldots, X_{n}$, suppose we cannot find suitable candidates for $x_{n+1}$ and $X_{n+1}$. Then for every 
$x \in X_{n}$ and every subspace $Y$ of $X_{n}$ we can find a subspace $Z \subset Y$ such that either

(a) $\rho_{\Delta_{n+1}} \cap\left[x_{1}, \ldots, x_{n}, x ; Z\right]$ is empty

or

(b) $\rho_{\Gamma_{n+1}}$ is strategically large for $\left[x_{1}, \ldots, x_{n}, x ; Z\right]$.

Let $\Pi$ be the set of singleton $*$-pairs $(x, Z)$ with $x \in X_{n}$ and $Z \subset X_{n}$ such that either (a) or (b) holds. We shall apply Corollary 2.3. Condition (ii) of this corollary is obvious, and we have just shown that condition (i) holds as well. Applying the corollary with $\delta=\delta_{n+1}$, we obtain a subspace $Y \subset X_{n+1}$ such that, for every $*$-pair $(y, Z)$ with $y \in Y$ and $Z \subset Y$, there exists $x$ with $\|y-x\| \leqslant \delta_{n+1}$ such that either (a) or (b) holds for the pair $(x, Z)$. Since $\|y-x\| \leqslant \delta_{n+1}$, the first alternative implies that $\rho_{\Delta_{n}} \cap\left[x_{1}, \ldots, x_{n}, y ; Z\right]$ is empty and the second implies that $\rho_{\Gamma_{n}}$ is strategically large for $\left[x_{1}, \ldots, x_{n}, y ; Z\right]$. In particular, one of these two conclusions is true when $Z=Y$. (There is a small technical point which is that $(y, Y)$ is not a $*$-pair. However, the conclusion can be seen by considering for each $y \in Y$ the $*$-pair $(y, Z)$, where $Z=\{z \in Y: y<z\}$.)

The set of $y$ such that $\rho_{\Delta_{n}} \cap\left[x_{1}, \ldots, x_{n}, y ; Y\right]$ is empty cannot contain a subspace $Z$ of $Y$ since then $\rho_{\Delta_{n}} \cap\left[x_{1}, \ldots, x_{n} ; Z\right]$ is empty, which contradicts (ii) of our inductive hypothesis. Therefore, the set of $y$ such that $\rho_{\Gamma_{n}}$ is strategically large for $\left[x_{1}, \ldots, x_{n}, y ; Y\right]$ is large for $Y$. But this gives $\mathrm{P}$ a winning strategy for the game $\rho_{\Gamma_{n}}\left[x_{1}, \ldots, x_{n} ; Y\right]$ and contradicts (iii) of our inductive hypothesis.

We now claim that the subspace generated by $\left(x_{n}\right)_{n=1}^{\infty}$ has empty intersection with $\sigma_{-\Theta}$. Indeed, let $\left(z_{1}, \ldots, z_{k}\right) \in \Sigma_{f}$ be a sequence contained in the subspace $\left\langle x_{1}, \ldots, x_{n}\right\rangle$. By property (ii), the sequence $\left(x_{1}, \ldots, x_{n}\right)$ can be extended to a sequence $\left(x_{1}, \ldots, x_{m}\right)$ in $\rho_{\Delta}$. Choose $\left(x_{1}^{\prime}, \ldots, x_{m}^{\prime}\right) \in \rho$ such that $\left\|x_{i}-x_{i}^{\prime}\right\| \leqslant \delta_{i}$ for every $i \leqslant m$. Let $\left(z_{1}^{\prime}, \ldots, z_{k}^{\prime}\right)$ be the corresponding perturbation of $\left(z_{1}, \ldots, z_{k}\right)$, and notice that the minimality condition satisfied by $\rho$ ensures that $\left(z_{1}^{\prime}, \ldots, z_{k}^{\prime}\right) \notin \sigma$. Our choice of $\Delta$ guarantees that $\left\|z_{j}-z_{j}^{\prime}\right\| \leqslant \Theta_{j}$ for every $j \leqslant k$. But this proves that $\left(z_{1}, \ldots, z_{k}\right) \notin \sigma_{-\Theta}$ and our claim is proved. This contradicts the assumption that $\sigma_{-\Theta}$ was large.

It is not hard to see that the above result is equivalent to Theorem 2.1.

Proof of Theorem 2.1. Let $\tau=\sigma_{\Delta / 2}$. The assumption of the theorem and the fact that $\sigma \subset \tau_{-\Delta / 2}$ tell us that $\tau_{-\Delta / 2}$ is large for $X$. Therefore, applying Theorem 3.1 to $\tau$ (with $\Theta$ replaced by $\Delta / 2$ ) we can find some positive real sequence $\Gamma \leqslant \Delta / 2$ such that $\tau_{\Gamma}$ is strategically large for some subspace of $X$. But $\tau_{\Gamma} \subset \sigma_{\Delta}$, so the result is proved. 
Before we apply Theorem 2.1, let us introduce two further definitions. We shall say that a block basis $\left(x_{n}\right)_{n=1}^{\infty}$ is $C$-unconditional if it generates a subspace which contains no $C$-conditional finite sequences of blocks. $(C$-conditional sequences were defined just after Lemma 1.7.) We shall say that a Banach space $X$ is $C$-hereditarily indecomposable if for every pair $Y, Z$ of block subspaces of $X$ we can find $y \in Y$ and $z \in Z$ such that $\|y+z\|>C\|y-z\|$. This is equivalent to condition (ii) of Lemma 1.7 for that particular value of $C$. Lemma 1.7 asserts that $X$ is hereditarily indecomposable if and only if it is $C$-hereditarily indecomposable for every $C$.

Corollary 3.2. Let $X$ be a Banach space. Then either $X$ contains a $C$-unconditional block basis or for every $\varepsilon>0$ it has a $(C-\varepsilon)$-hereditarily indecomposable block subspace.

Proof. Let $\sigma$ be the set of all sequences $\left(x_{1}, \ldots, x_{n}\right) \in \Sigma_{f}$ that are $C$-conditional and contain at least one vector $x_{i}$ of norm 1 . If $X$ contains no $C$-unconditional block basis, then $\sigma$ is large, since every subspace contains a $C$-conditional sequence $\left(y_{1}, \ldots, y_{n}\right)$, and if we divide this by the largest value of $\left\|y_{i}\right\|$ we obtain a sequence in $\sigma$. Therefore, by Theorem 2.1 , for any $\Delta>0$ we can find a block subspace $W$ of $X$ such that $\sigma_{\Delta}$ is strategically large for $W$. Let us choose $\Delta$ such that $\sum_{i=1}^{\infty} \delta_{i}=\eta$ for some $\eta>0$ satisfying the inequality $(1+2 \eta)^{-1}(C-2 \eta) \geqslant C-\varepsilon$.

Now let $Y$ and $Z$ be arbitrary block subspaces of $W$ and consider the strategy for S, the subspace player, of alternating $Y$ and $Z$. Since $\sigma_{\Delta}$ is strategically large for $W, \mathrm{P}$ can defeat this strategy, which means that $\mathrm{P}$ can choose a sequence $\left(y_{1}, z_{1}, y_{2}, z_{2}, \ldots, y_{n}, z_{n}\right) \in \sigma_{\Delta}$ such that $y_{i}$ belongs to $Y$ and $z_{i}$ belongs to $Z$ for every $i$. (An unimportant technical point is that P's strategy may succeed after an odd number of moves. One can either alter the statement of Lemma 1.7 or let $\mathrm{P}$ choose a sufficiently small $z_{n}$ to finish. The second approach is possible because of the strict inequality in the definition of $C$-conditional sequences.) We can then find $\left(y_{1}^{\prime}, z_{1}^{\prime}, \ldots, y_{n}^{\prime}, z_{n}^{\prime}\right) \in \sigma$ such that $\left\|y_{i}-y_{i}^{\prime}\right\| \leqslant \delta_{2 i-1}$ and $\left\|z_{i}-z_{i}^{\prime}\right\| \leqslant \delta_{2 i}$ for every $i \leqslant n$. Since the basis of $X$ is monotone, the norms $\left\|\sum_{i=1}^{n}\left(y_{i}^{\prime}+z_{i}^{\prime}\right)\right\|$ and $\left\|\sum_{i=1}^{n}\left(y_{i}^{\prime}-z_{i}^{\prime}\right)\right\|$ are both at least $1 / 2$. We therefore know that

$$
\left\|\sum_{i=1}^{n}\left(y_{i}^{\prime}+z_{i}^{\prime}\right)\right\|>C\left\|\sum_{i=1}^{n}\left(y_{i}^{\prime}-z_{i}^{\prime}\right)\right\| \geqslant C / 2 .
$$

By the triangle inequality and our choice of $\Delta$, we know that

$$
\left\|\sum_{i=1}^{n}\left(y_{i}+z_{i}\right)\right\| \geqslant\left\|\sum_{i=1}^{n}\left(y_{i}^{\prime}+z_{i}^{\prime}\right)\right\|-\eta
$$


and

$$
\left\|\sum_{i=1}^{n}\left(y_{i}-z_{i}\right)\right\| \leqslant\left\|\sum_{i=1}^{n}\left(y_{i}^{\prime}-z_{i}^{\prime}\right)\right\|+\eta .
$$

It follows from our choice of $\eta$ that

$$
\left\|\sum_{i=1}^{n}\left(y_{i}+z_{i}\right)\right\|>(C-\varepsilon)\left\|\sum_{i=1}^{n}\left(y_{i}-z_{i}\right)\right\| .
$$

Since $Y$ and $Z$ were arbitrary subspaces of $W$, we have shown that $W$ is $(C-\varepsilon)$-hereditarily indecomposable.

A simple diagonalization now completes the proof of Theorem 1.4.

Proof of Theorem 1.4. If $X$ has no subspace with an unconditional basis, then Lemma 1.6 tells us that, for every $C$, every block subspace of $X$ contains a $C$-conditional block sequence. By Corollary 3.2 we can therefore find a nested sequence $W_{1} \supset W_{2} \supset \ldots$ of block subspaces of $X$ such that, for every $n$, the subspace $W_{n}$ is $n$-hereditarily indecomposable. Let $\left(w_{n}\right)_{n=1}^{\infty}$ be a block basis of $X$ such that $w_{n} \in W_{n}$ for every $n$ and let $W$ be the subspace generated by $\left(w_{n}\right)_{n=1}^{\infty}$. We claim that $W$ is hereditarily indecomposable. To see this, let $Y$ and $Z$ be arbitrary block subspaces of $W$ and let $C$ be any real number. Choose a positive integer $n \geqslant C$. Then $Y \cap W_{n}$ and $Z \cap W_{n}$ are infinite-dimensional. Since $W_{n}$ is $n$-hereditarily indecomposable, we can find a sequence satisfying condition (ii) of Lemma 1.7 for this particular $C$. But $C$ was arbitrary, so the condition holds in general. Lemma 1.7 therefore implies that $W$ is hereditarily indecomposable, as claimed.

\section{Definitions and preliminary results for infinite sequences}

We begin this section with a brief discussion of the connections between our results so far and known results of infinite Ramsey theory. The statement of Theorem 2.1 is strongly reminiscent of a result in infinite Ramsey theory due to Nash-Williams $[\mathrm{N}-\mathrm{W}]$, which says the following. Let $\mathbb{N}^{\omega}$ be the set of all infinite subsets of $\mathbb{N}$. If $A$ is an open subset of $\mathbb{N}^{\omega}$ (in the product topology) then either $A$ or its complement contains all infinite subsets of some $X \in \mathbb{N}^{\omega}$. A set $A$ with this property is called a Ramsey set.

It is easy to check that Nash-Williams's result is equivalent to the following statement. Let $\mathbb{N}<\omega$ be the set of all finite subsets of $\mathbb{N}$ and let $A \subset \mathbb{N}<\omega$. Then there is an infinite subset $X$ of $\mathbb{N}$ such that either no finite subset of $X$ is in $A$ or for every infinite subset $Y$ of $X$ there exists $n$ such that $Y \cap\{1,2, \ldots, n\} \in A$. Notice that if the first alternative does not hold, then $A$ is large in an obvious sense. So Nash-Williams's theorem asserts that if $A$ is a large subset of $\mathbb{N}<\omega$ 
then there is an infinite subset $X$ of $\mathbb{N}$ for which $A$ has a much stronger largeness property. This formulation makes the resemblance with Theorem 2.1 very clear.

Nash-Williams's theorem was extended to all Borel sets by Galvin and Prikry [GP]. A combinatorial lemma of theirs inspired our proof of Theorem 2.1. Silver [S] proved that all analytic sets are Ramsey, and Mathias proved that in a model constructed by Solovay all sets are Ramsey. From these proofs there emerged a natural strengthening of the Ramsey property, which was shown by Ellentuck [E] to be equivalent to the property of Baire in a certain topology. For proofs of the Galvin-Prikry lemma and Ellentuck's theorem, see $[\mathrm{Bo}]$ and for further results in this direction, see $[\mathrm{M}]$.

The main aim of the next two sections is to extend Theorem 2.1 in a similar way. However, as we will explain later (see the appendix), the obvious analogue of Ellentuck's characterization is false, so we must be satisfied with a result more like Silver's. In order to state it, we shall need some definitions concerning infinite sequences and sets of infinite sequences.

The obvious way to extend our earlier results would be to redefine $\Sigma(X)$ as the set of all infinite sequences $x_{1}<x_{2}<\ldots$ of nonzero vectors in $X$ such that $\left\|x_{n}\right\| \leqslant 1$ for every $n$. Instead, for technical reasons, our definition will be slightly different: let $\Sigma(X)$ be the set of all sequences of pairs $\left(x_{1}, \lambda_{1}\right),\left(x_{2}, \lambda_{2}\right), \ldots$ where $x_{1}<x_{2}<\ldots$ are vectors of norm 1 , and $\lambda_{1}, \lambda_{2}, \ldots$ are real numbers in the interval $[0,1]$. Of course, we can usually identify the pair $(x, \lambda)$ with the vector $\lambda x$. The main difference between our definition and the obvious definition is that if $x \neq y$, then we distinguish between the pairs $(x, 0)$ and $(y, 0)$. In order to save writing, we shall usually use a single letter to denote one of these pairs, unless it is important to be careful. We shall refer to elements of $\Sigma$ as a block bases. Sometimes we shall discuss finite block bases. Let us now redefine $\Sigma_{f}$ to be the set of finite sequences $\left(\left(x_{1}, \lambda_{1}\right), \ldots,\left(x_{n}, \lambda_{n}\right)\right)$ with the $\left(x_{i}, \lambda_{i}\right)$ as above and $x_{1}<\ldots<x_{n}$. The support of a pair $\left(x_{n}, \lambda_{n}\right)$ is defined to be the support of the vector $x_{n}$. The subspace generated by the pairs ) $\left(x_{1}, \lambda_{1}\right),\left(x_{2}, \lambda_{2}\right), \ldots$ is defined to be the (block) subspace generated by the vectors $x_{1}, x_{2}, \ldots$

Several definitions to do with finite sequences can be easily adapted for infinite sequences. For example, if $X$ is a Banach space, $Y$ is a block subspace of $X$ and $A=\left(y_{1}, \ldots, y_{m}\right)$ is a finite sequence of blocks (that is, pairs of the above kind) then $[A ; Y]$ is now defined as the set of all infinite sequences $\left(z_{n}\right)_{n=1}^{\infty} \in \Sigma$ such that $z_{i}=y_{i}$ for $i \leqslant m$ and $z_{i} \in Y$ for $i>m$. Again we denote this by $[Y]$ if $A$ is the null sequence. If $\sigma$ is a subset of $\Sigma$, then we write $\sigma[A ; Y]$ for the set of all sequences $\left(w_{n}\right)_{n=1}^{\infty} \in \Sigma(Y)$ such that $\left(y_{1}, \ldots, y_{m}, w_{1}, w_{2}, \ldots\right) \in \sigma$. We say that $\sigma$ is large for $[A ; Y]$ if every block subspace of $Y$ contains a sequence in $\sigma[A ; Y]$. Once again, $\sigma$ is large for $[A ; Y]$ if and only if $\sigma[A ; Y]$ is large for $Y$. 
Given a subset $\sigma$ of $\Sigma$, we can define an infinite game just as we did for finite sequences. The only difference is that P's aim is to produce an infinite sequence that lies in $\sigma$, so the game always lasts for ever (although one player may have a guaranteed win after finite time regardless of future moves). We say that $\sigma$ is strategically large for $[A ; Y]$ if $\mathrm{P}$ has a winning strategy for the set $\sigma[A ; Y]$ when all of S's moves are required to be subspaces of $Y$.

Finally, we consider two topologies on $\Sigma$. Most of the time it will be convenient to take as basic open sets all sets of the form $\left\{\left(x_{n}\right)_{n=1}^{\infty}: x_{n}=\right.$ $y_{n}$ for $\left.1 \leqslant n \leqslant N\right\}$. In other words, we put the discrete topology on $X$ and then take the product topology on $\Sigma(X)$. However, for our main result we take a different topology. First, define a metric on $S(X) \times[0,1]$ by $d((x, \lambda),(y, \mu))=$ $\|x-y\|+|\lambda-\mu| .(S(X)$ is the unit sphere of $X$. Roughly speaking, we have made a hole where zero used to be.) From this we derive a topology on $X$ and hence a different product topology on $\Sigma(X)$. A basic open neighbourhood of the sequence $\left(\left(x_{n}, \lambda_{n}\right)\right)_{n=1}^{\infty}$ is now a set of the form

$$
\left\{\left(\left(y_{n}, \mu_{n}\right)\right)_{n=1}^{\infty}:\left\|y_{i}-x_{i}\right\|+\left|\mu_{i}-\lambda_{i}\right|<\varepsilon_{i} \text { for every } i \leqslant N\right\}
$$

for some positive integer $N$ and positive real sequence $\left(\varepsilon_{1}, \ldots, \varepsilon_{N}\right)$. The advantage of the first topology is that it is less messy to talk about open and closed sets, and the advantage of the second is that it makes $\Sigma(X)$ a Polish space (see Lemma 4.2 below), which is more convenient for talking about analytic sets. (It is in order to make $\Sigma$ complete metrizable that we allow "zero vectors" in a block basis.) However, a perturbation is involved in our result, so the distinction between the two topologies is not at all important for applications. We shall refer to D-open and N-open sets and so on (for "discrete" and "norm") when it is not otherwise clear which topology is meant.

We have not yet defined perturbations of infinite sequences. If $\sigma$ is a subset of $\Sigma(X)$ and $\Delta>0$ is an infinite sequence of positive real numbers, then let $\sigma_{\Delta}$ denote the set of all sequences $\left(x_{n}\right)_{n=1}^{\infty}$ such that there exists a sequence $\left(y_{n}\right)_{n=1}^{\infty} \in \sigma$ with $d\left(x_{n}, y_{n}\right) \leqslant \delta_{n}$ for every $n$. (Note that the $x_{n}$ and $y_{n}$ are elements of $S(X) \times[0,1]$ and $d$ is the metric defined above.) We now have enough notation to state the main theorem of this and the next section.

Theorem 4.1. Let $X$ be a Banach space, let $\sigma \subset \Sigma(X)$ be $\mathrm{N}$-analytic and large for $X$ and let $\Delta>0$. Then there is a subspace $Y$ of $X$ such that $\sigma_{\Delta}$ is strategically large for $Y$.

Let us give a definition which will be useful for the rest of the paper.

Definition. A set $\sigma \subset \Sigma(X)$ is weakly Ramsey if for every $\Delta>0$ there is a subspace $Y \subset X$ such that either $\sigma \cap[Y]$ is empty or $\sigma_{\Delta}$ is strategically large for $Y$. 
Thus, Theorem 4.1 states that $\mathrm{N}$-analytic sets are weakly Ramsey, and a set $\sigma \subset \Sigma(X)$ is weakly Ramsey if the conclusion of Theorem 4.1 holds for $\sigma$.

For the rest of this section we shall prove that D-open sets and N-closed sets are weakly Ramsey, and then we shall prove a few lemmas which are needed for Theorem 4.1. First, we check that Theorem 2.1 still holds now that we have redefined $\Sigma_{f}$. One way of doing this is simply to check that the proof we gave is still valid under the new interpretation. However, it may reassure the reader to see that one can deduce the result for the new $\Sigma_{f}$ from Theorem 2.1.

Lemma 4.2. Let $X$ be a Banach space, let $\sigma$ be any subset of $\Sigma_{f}(X)$ and let $\Delta$ be a sequence of positive real numbers. Then $X$ has a subspace $Y$ such that either $\sigma[Y]=\emptyset$ or $P$ has a winning strategy for the game $\sigma_{\Delta}[Y]$.

Proof. Define $\rho$ to be the set of all sequences

$$
\left(\left(1+\lambda_{1}\right) x_{1} / 2, \ldots,\left(1+\lambda_{n}\right) x_{n} / 2\right)
$$

such that the sequence

$$
\left(\left(x_{1}, \lambda_{1}\right), \ldots,\left(x_{n}, \lambda_{n}\right)\right)
$$

is in $\sigma$. It follows from the triangle inequality that

$$
\|(1+\lambda) x-(1+\mu) y\| \geqslant \max \{|\lambda-\mu|,\|x-y\|-|\lambda-\mu|\} .
$$

Therefore, applying Theorem 2.1 to $\rho$ with $\Delta$ replaced by $\Delta / 4$ we obtain the desired conclusion.

Theorem 4.3. 4.3. All D-open sets are weakly Ramsey.

Proof. This is just a reformulation of Lemma 4.2. Let $\sigma$ be a D-open set. Let $\sigma_{f}$ be the set of finite sequences $\left(x_{1}, \ldots, x_{n}\right) \in \Sigma_{f}$ such that all their extensions to sequences in $\Sigma$ are elements of $\sigma$. Since $\sigma$ is a D-open set, every sequence in $\sigma$ has an initial segment belonging to $\sigma_{f}$. If $\sigma$ is large for $X$, it follows that $\sigma_{f}$ is large for $X$ as well. Therefore, by Lemma 4.2, for every $\Delta>0$ there is a subspace $Y$ of $X$ such that $\left(\sigma_{f}\right)_{\Delta}$ is strategically large for $Y$. If $\mathrm{P}$ uses this strategy and plays for ever, then the resulting infinite sequence belongs to $\sigma_{\Delta}$. Thus, $\sigma_{\Delta}$ is also strategically large for $Y$. This shows that $\sigma$ is weakly Ramsey, as claimed.

Although it is weaker than our main result and not needed for the proof, we shall now show that N-closed sets are weakly Ramsey, since the proof is quite short, and we shall apply this weaker result directly in $\S 7$. As a matter of fact, we prove slightly more, by defining a finer topology, which we shall call the D-*-topology, very similar to the so-called *-topology,or Mathias topology, 
on the infinite subsets of $\mathbb{N}$. We shall then show that D-*-closed sets are close to being weakly Ramsey and that N-closed sets are genuinely weakly Ramsey. In Section 7, we shall apply this result to obtain a second Banach space dichotomy.

The $\mathrm{D}$-*-topology is the topology generated by the sets $[A ; Y]$ defined at the beginning of this section. Thus, a basic open neighbourhood of a sequence $\left(x_{n}\right)_{n=1}^{\infty}$ is some set of sequences $[A ; Y]$, where $A$ is an initial segment $\left(x_{1}, \ldots, x_{m}\right)$ and $Y$ is a block subspace containing $x_{i}$ for every $i>m$. (Similarly, one can define the $\mathrm{N}$-*-topology to have basic open sets of the following form: the set of all sequences $\left(x_{n}\right)_{n=1}^{\infty} \in \Sigma$ such that $\left\|x_{i}-y_{i}\right\|<\delta$ for $i \leqslant n$ and $x_{i} \in Y$ for $i>n$, where $\delta>0, y_{1}<\ldots<y_{n}$ and $Y$ is an infinite-dimensional block subspace.) A set $\sigma$ is completely weakly Ramsey if, whenever $\sigma$ is large in $[A ; Y]$ and $\Delta>0$, there is a subspace $Z$ of $Y$ such that $\mathrm{P}$ has a winning strategy for the game $\sigma_{\Delta}[A ; Z]$. Note that if every analytic set is weakly Ramsey, then every analytic set is completely weakly Ramsey. We shall show in the appendix that the natural analogue of Ellentuck's theorem, that a set is completely weakly Ramsey if and only if it is a Baire set in the N-*-topology, is false. In fact, we show that the intersection of two completely weakly Ramsey sets need not be completely weakly Ramsey.

Given $A$ and $Y$ as above, we shall follow Galvin and Prikry by saying that $Y$ accepts $A$ (into $\sigma$ ) if $[A ; Y] \subset \sigma$, and that $Y$ rejects $A$ (from $\sigma$ ) if no subspace $Z \subset Y$ accepts $A$. Saying that $Y$ rejects the null sequence from the complement of $\sigma$ is equivalent to saying that every block subspace $Z \subset Y$ contains a sequence in $\sigma$, or in other words that $\sigma$ is large in $Y$.

We are about to apply Corollary 2.4 several times. However, strictly speaking one needs a different result concerning modified blocks $(x, \lambda)$. Such a result can easily be proved in an identical way, so we shall simply apply Corollary 2.4, interpreting the vectors there as pairs $(x, \lambda) \in S(X) \times[0,1]$.

Lemma 4.4. Let $\sigma \subset \Sigma$ be a set of infinite sequences and let $\Delta_{0}>\Delta_{1}>$ $\Delta_{2}>\ldots$ be a sequence of positive real sequences. Then there is a subspace $Y \subset X$ such that, for any $k$ and any sequence $\left(y_{1}, \ldots, y_{k}\right) \in \Sigma_{f}(Y)$, either $Y$ accepts $y_{1}, \ldots, y_{k}$ into $\sigma_{\Delta_{k-1}}$ or $Y$ rejects $y_{1}, \ldots, y_{k}$ from $\sigma_{\Delta_{k}}$.

Proof. For each $k$ let $\Gamma_{k}=\frac{1}{2}\left(\Delta_{k-1}+\Delta_{k}\right)$ and let $\Theta_{k}=\frac{1}{2}\left(\Delta_{k-1}-\Delta_{k}\right)$. Let $\Pi_{k}$ be the set of pairs $(A, Z)$ such that $A$ is a sequence of length $k$ and $Z$ either accepts $A$ into $\sigma_{\Gamma_{k}}$ or rejects $A$ from $\sigma_{\Gamma_{k}}$. It is easy to see that the conditions of Corollary 2.4 hold, so we can pass to a subspace such that, for every $k$ and every $A$ of size $k$, every pair $(A, Z)$ is in $\left(\Pi_{k}\right)_{\Theta_{k}}$. Given any pair $(A, Z)$ with $A$ of length $k$, choose $A^{\prime} \in \Sigma_{f}$ such that $d\left(A, A^{\prime}\right) \leqslant \Theta_{k}$ and $\left(A^{\prime}, Z\right) \in \Pi_{k}$. If $Z$ accepts $A^{\prime}$ into $\sigma_{\Gamma_{k}}$, then $Z$ accepts $A$ into $\sigma_{\Delta_{k-1}}$ and if $Z$ rejects $A^{\prime}$ from $\sigma_{\Gamma_{k}}$ then $Z$ rejects $A$ from $\sigma_{\Delta_{k}}$. This proves the result. 
TheOREM 4.5. Let $\sigma$ be a $\mathrm{D}$-*-closed subset of $\Sigma$, let $\tau$ be the complement of $\sigma$ and let $\Delta>0$. Then there exists a subspace $Y \subset X$ such that either $P$ has a winning strategy for the game $\sigma[Y]$ or $[Y] \subset \tau_{\Delta}$.

Proof. Suppose there is no subspace $Y$ such that $[Y] \subset \tau_{\Delta}$. Then $X$ rejects $\emptyset$ from $\tau_{\Delta}$. For $k=0,1,2, \ldots$ let $\Delta_{k}=2^{-k} \Delta$. By Lemma 4.4, we may assume that, for every sequence $\left(x_{1}, \ldots, x_{n}\right), X$ either accepts $\left(x_{1}, \ldots, x_{n}\right)$ into $\tau_{\Delta_{n-1}}$ or rejects it from $\tau_{\Delta_{n}}$.

Now let $\mathrm{P}$ play the following strategy: for the $n^{\text {th }}$ move, choose $x_{n}$ such that $X$ rejects $\left(x_{1}, \ldots, x_{n}\right)$ from $\tau_{\Delta_{n}}$. We shall show both that this is possible and that it is a winning strategy.

Suppose then that $\mathrm{P}$ has played the strategy successfully for $n$ moves. We know that $X$ rejects $\left(x_{1}, \ldots, x_{n}\right)$ from $\tau_{\Delta_{n}}$. Now let $\mathrm{S}$ play the subspace $X_{n+1}$. If $\mathrm{P}$ cannot continue the strategy, then, for every $x \in X_{n+1}, X$ does not reject $\left(x_{1}, \ldots, x_{n}, x\right)$ from $\tau_{\Delta_{n+1}}$. But because of our assumption about $X$, this implies that $X$ accepts $\left(x_{1}, \ldots, x_{n}, x\right)$ into $\tau_{\Delta_{n}}$ for every $x \in X_{n+1}$. It follows that $X_{n+1}$ accepts $\left(x_{1}, \ldots, x_{n}\right)$ into $\tau_{\Delta_{n}}$. But this contradicts the fact that $X$ rejects $\left(x_{1}, \ldots, x_{n}\right)$ from $\tau_{\Delta_{n}}$.

We have shown that the strategy is possible. Now let $\left(x_{1}, x_{2}, \ldots\right)$ be a sequence produced by B when using it. Then we certainly know that, for every $n, X$ rejects $\left(x_{1}, \ldots, x_{n}\right)$ from $\tau$. That means that, given any subspace $Y \subset X$, $\left[x_{1}, \ldots, x_{n} ; Y\right] \cap \sigma \neq \emptyset$. Since $\sigma$ is $*$-closed, it follows that $\left(x_{1}, x_{2}, \ldots\right) \in \sigma$.

Because of the way we defined weakly Ramsey sets, Theorem 4.5 does not imply that D-*-closed sets are weakly Ramsey, although this is true up to an arbitrarily small perturbation. Moreover, if $\sigma$ is $\mathrm{D}-*$-closed, it does not follow that $\sigma_{\Delta}$ is $\mathrm{D}$-*-closed. Although it is not important for applications, we shall now show that N-closed sets are weakly Ramsey, using the following simple result.

Lemma 4.6. Let $\sigma$ be $\mathrm{N}$-closed and let $\Delta>0$. Then $\sigma_{\Delta}$ is also $\mathrm{N}$-closed.

Proof. Since $\sigma_{\Delta}=\left(\sigma_{\Delta / 2}\right)_{\Delta / 2}$ we can assume that every $\delta_{n}$ is at most $1 / 2$. We shall use bold face letters to denote sequences in $\Sigma$. Let $\mathbf{x}$ belong to the closure of $\sigma_{\Delta}$. Then there exist sequences $\mathbf{y}^{m} \in \sigma_{\Delta}$ converging pointwise to $\mathbf{x}$. For every $m$ we can find a sequence $\mathbf{z}^{m} \in \sigma$ such that $d\left(\mathbf{y}^{m}, \mathbf{z}^{m}\right) \leqslant \Delta$. For fixed $n$, the vectors $z_{n}^{m}$ (or strictly speaking elements of $S(X) \times[0,1]$ ) have bounded support, as otherwise $d\left(z_{n+1}^{m}, x_{n+1}^{m}\right)$ would be at least $2 / 3$ for sufficiently large $m$. Therefore we can find a sequence $m_{1}, m_{2}, \ldots$ such that $\mathbf{z}^{m_{i}}$ converges pointwise, to $\mathbf{z}$, say. Since $\sigma$ is N-closed, $\mathbf{z}$ must be in $\sigma$. Because $\mathbf{z}^{m} \rightarrow \mathbf{z}$ and $\mathbf{y}^{m} \rightarrow \mathbf{x}$ pointwise we must have $d\left(z_{n}, x_{n}\right) \leqslant \delta_{n}$ for every $n$, which proves that $\mathbf{x} \in \sigma_{\Delta}$, as required.

Corollary 4.7. Every N-closed set is weakly Ramsey. 
Proof. Let $\sigma$ be N-closed and let $\Delta>0$. Then $\sigma_{\Delta / 2}$ is N-closed, by Lemma 4.6, and in particular D-*-closed. Applying Theorem 4.5 to $\sigma_{\Delta / 2}$ with $\Delta / 2$ replacing $\Delta$ we obtain the result.

The rest of this section is devoted to lemmas which will be used to prove our main result, Theorem 4.1. If $\sigma \subset \Sigma$ has the property that it is not large for any $[A ; Y]$, then we shall say that it is nowhere large, and otherwise that it is large somewhere. In the usual infinite Ramsey theorems, it is shown that being nowhere large is the same as being $*$-nowhere dense. That is false here (see the appendix). We do, however, have the following useful lemma, which states that the nowhere large sets are, up to perturbation, closed under countable unions.

LEMmA 4.8. Let $\sigma=\bigcup_{n=1}^{\infty} \sigma_{n}$, let $\Delta_{n}>0$ for every $n$ and suppose that $\left(\sigma_{n}\right)_{\Delta_{n}}$ is nowhere large for every $n$. Then $\sigma$ is nowhere large.

Proof. Let $(B, W)$ be any pair. We must show that $\sigma$ is not large for $[B ; W]$. For each $n$ let $\Pi_{n}$ be the set of pairs $(A, Z)$ in $W$ such that $A$ is a sequence of length exactly $n$ and $\left(\sigma_{n}\right)_{\Delta_{n}} \cap[(B, A) ; Z]$ is empty. (We mean by $(B, A)$ the concatenation of $A$ and $B$.) Our hypothesis implies that the sets $\Pi_{n}$ satisfy the conditions for Corollary 2.4. We can therefore find a subspace $Y \subset W$ such that $(A, Z) \in\left(\Pi_{n}\right)_{\Delta_{n}}$ for every pair $(A, Z)$ in $Y$ with $A$ of length $n$. It is easy to see that this implies that $[(B, A) ; Z] \cap \sigma_{n}=\emptyset$ for every such pair. This says that no sequence $C$ of length $n$ in $Y$ can be added to the end of $B$ and then extended in $Y$ to a sequence in $\sigma_{n}$. It follows that $[B ; Y] \cap \sigma=\emptyset$, because if we could find $D \in \Sigma(Y)$ and $n$ such that $(B, D) \in \sigma_{n}$ then the first $n$ terms of $D$ would give us a sequence $C$ contradicting what we have just established. Since $B$ and $W$ were arbitrary, we have shown that $\sigma$ is nowhere large.

For technical reasons we need a slight extension of Lemma 4.8 (Corollary 4.10 below).

Lemma 4.9. If $\sigma$ is large for $[B ; Y]$ and $\Delta>0$, then for every $m \in \mathbb{N}$ there exists a sequence $A$ of length $m$ in $Y$ and a subspace $Z \subset Y$ such that $\sigma_{\Delta}$ is large in $[(B, A) ; Z]$.

Proof. Suppose $\sigma$ is a counterexample to the assertion. Let $\Pi_{m}$ be the set of pairs $(A, Z)$ in $Y$ such that $A$ is a sequence of length $m$ and $\sigma_{\Delta} \cap[(B, A) ; Z]=$ $\emptyset$. For $n \neq m$ let $\Pi_{n}$ be the set of all pairs. Our assumption implies that the $\Pi_{n}$ satisfy the conditions of Corollary 2.4. Therefore we can find a subspace $W \subset Y$ such that $(A, Z) \in \Pi_{m}$ for every pair $(A, Z)$ in $W$ with $A$ of length $m$, 
which implies that $\sigma \cap[(B, A) ; Z]=\emptyset$ for every such pair. But this says that no sequence from $W$ of length $m$ can be put on to the end of $B$ and extended in $W$ to a sequence in $\sigma$. Therefore $\sigma$ is not large for $[B ; Y]$.

Corollary 4.10. If $\sigma$ is large somewhere, $\sigma=\bigcup_{n=1}^{\infty} \sigma_{n}, \Delta_{1}, \Delta_{2}, \ldots$ are positive real sequences and $k_{1}, k_{2}, \ldots$ is a sequence of integers, then there exists $n$ such that $\left(\sigma_{n}\right)_{\Delta_{n}}$ is large for some $[A ; Z]$ with $A$ a sequence of size at least $k_{n}$.

Proof. By Lemma 4.8 we can find $n$ and a pair $(B, Y)$ such that $\sigma_{\Delta_{n} / 2}$ is large for $[B ; Y]$. Then by Lemma 4.9 with $m=k_{n}, \sigma=\sigma_{\Delta_{n} / 2}$ and $\Delta=\Delta_{n} / 2$, we can find a sequence $C \in \Sigma_{f}$ of length $k_{n}$ and a subspace $Z \subset Y$ such that $\sigma_{\Delta_{n}}$ is large in $[B, C ; Z]$. The length of the sequence $(B, C)$ is certainly at least $k_{n}$ so we are done.

The next lemma is very similar in spirit to Lemma 4.4 .

Lemma 4.11. Let $X$ be a Banach space, let $\sigma_{1}, \sigma_{2}, \ldots$ be a sequence of subsets of $\Sigma(X)$ and let $\Delta_{1}, \Delta_{2}, \ldots$ be a sequence of positive real sequences. Then there exists a subspace $Y \subset X$ such that, for every $n$ and every sequence $A \in \Sigma_{f}(Y)$ of length at least $n$, either $\sigma_{n} \cap[A ; Y]$ is empty or $\left(\sigma_{n}\right)_{\Delta_{n}}$ is large for $[A ; Y]$.

Proof. For each $n$ let $\Pi_{n}$ be the set of all $*$-pairs $(A, Z)$ such that either $\left(\sigma_{n}\right)_{\Delta_{n} / 2} \cap[A ; Z]$ is empty or $\left(\sigma_{n}\right)_{\Delta_{n} / 2}$ is large for $[A ; Z]$. It is very easy to check that the sets $\Pi_{n}$ satisfy the conditions of Lemma 2.2. Therefore, there is a subspace $Y$ such that every *-pair $(A, Z)$ in $Y$ with $A$ of length at least $n$ belongs to $\left(\Pi_{n}\right)_{\Delta_{n} / 2}$. This says that there is some $A^{\prime}$ with $d\left(A, A^{\prime}\right) \leqslant \Delta_{n} / 2$ with $\left(A^{\prime}, Z\right) \in \Pi_{n}$. If $\left(\sigma_{n}\right)_{\Delta_{n} / 2} \cap\left[A^{\prime}, Z\right]=\emptyset$ then $\sigma_{n} \cap[A ; Z]=\emptyset$. If $\left(\sigma_{n}\right)_{\Delta_{n} / 2}$ is large for $\left[A^{\prime} ; Z\right]$, then let $W$ be an arbitrary subset of $Z$. We can choose an infinite sequence $B \in \Sigma(W)$ such that $A<B$ and $\left(A^{\prime}, B\right) \in\left(\sigma_{n}\right)_{\Delta_{n} / 2}$. Then $(A, B) \in\left(\sigma_{n}\right)_{\Delta_{n}}$. This shows that $\left(\sigma_{n}\right)_{\Delta_{n}}$ is large for $[A ; Z]$. The proof is complete.

We end this section with a lemma of a more technical nature.

Lemma 4.12. Let $\sigma \subset \Sigma$ be large, let $\sigma=\bigcup_{n=1}^{\infty} \sigma_{n}$, let $\Delta>0$ be a real sequence and let $\psi$ be a function from $\mathbb{N}$ to $\mathbb{N}$. For any subspace $Y \subset X$ let $\rho(Y)$ be the set of sequences $B \in \Sigma_{f}(Y)$ such that there exists $n$ with $\max \{n, \psi(n)\}$ at most the size of $B$ and $\left(\sigma_{n}\right)_{\Delta}$ large for $[B ; Y]$. Then there exists a subspace $Y \subset X$ such that $\rho(Y)$ is strategically large for $Y$.

Proof. By Lemma 4.11 with each $\sigma_{n}$ replaced by $\left(\sigma_{n}\right)_{\Delta / 3}$ and each $\Delta_{n}$ equal to $\Delta / 3$, we can find a subspace $Z$ such that, for every $n \in \mathbb{N}$ and every sequence $A \in \Sigma_{f}(Z)$ of length at least $n$, either $\left(\sigma_{n}\right)_{\Delta / 3} \cap[A ; Z]$ is empty or $\left(\sigma_{n}\right)_{2 \Delta / 3}$ is large in $[A ; Z]$. 
Now $\sigma$ is large in $Z$ and therefore in any subspace of $Z$. Therefore, by Corollary 4.10, for any subspace $W \subset Z$ there exist $n$, a subspace $V \subset W$ and a sequence $B \in \Sigma_{f}(W)$ of length at least $\max \{n, \psi(n)\}$ such that $\left(\sigma_{n}\right)_{\Delta / 3}$ is large in $[B ; V]$. In particular, $\left(\sigma_{n}\right)_{\Delta / 3} \cap[B ; Z]$ is nonempty, so by our construction of $Z$ we know that $\left(\sigma_{n}\right)_{2 \Delta / 3}$ is large for $[B ; Z]$.

Define $\tau$ to be the set of sequences $B \in \Sigma_{f}(Z)$ such that there exists $n$ with $\max \{n, \psi(n)\}$ at most the length of $B$ and $\left(\sigma_{n}\right)_{2 \Delta / 3}$ large for $[B ; Z]$. We have just demonstrated that $\tau$ is large for $Z$. By Theorem 2.1 there is a subspace $Y \subset Z$ such that $\tau_{\Delta / 3}$ is strategically large for $Y$. The result follows, since $\tau_{\Delta / 3} \cap \Sigma_{f}(Y) \subset \rho(Y)$.

\section{Analytic sets are weakly Ramsey}

In preparation for the proof of Theorem 4.1, we recall the definition of and a basic fact about analytic sets. For a proof, and for general background in descriptive set theory, we recommend Chapter 7 of Jech's excellent book [J]. (This is the last chapter but the relevant part is very clear and more or less independent of the rest of the book.)

The Baire space $\mathcal{N}$ is the set $\mathbb{N}^{\mathbb{N}}$ with the product topology. Using continued fractions, one finds that $\mathcal{N}$ is homeomorphic to the set of irrational numbers. If $X$ is a topological space, an analytic subset of $X$ is the continuous image of a Borel subset. If $X$ is a Polish space, this can be shown to be equivalent to saying that the subset is a continuous image of $\mathcal{N}$. This equivalent definition is usually the easiest to use. It is clear that every Borel set is analytic. Souslin's theorem (which we do not need here) states that two disjoint analytic sets can be contained in two disjoint Borel sets, which implies that a set is Borel if and only if it and its complement are analytic.

Before giving the proof of Theorem 4.1, let us quickly verify that the set we shall be considering is indeed a Polish space.

Lemma 5.1. Let $X$ be a Banach space. Then $\Sigma(X)$ is a Polish space in the N-topology.

Proof. We must find a complete metric which gives rise to the N-topology on $\Sigma(X)$. Such a metric is given by

$$
d\left(\left(x_{n}, \lambda_{n}\right)_{n=1}^{\infty},\left(y_{n}, \mu_{n}\right)_{n=1}^{\infty}\right)=\max n^{-1} d\left(\left(x_{n}, \lambda_{n}\right),\left(y_{n}, \mu_{n}\right)\right)
$$

where on the right-hand side we have taken the metric defined earlier on $S(X) \times$ $[0,1]$. Let $S_{m}$ be the sequence $\left(\left(x_{m n}, \lambda_{m n}\right)\right)_{n=1}^{\infty}$ and suppose that the sequence $\left(S_{m}\right)_{m=1}^{\infty}$ is Cauchy in the above metric. The only small point to note is that the supports of the vectors $x_{m n}$ are bounded for fixed $n$, as otherwise the 
vectors $x_{m, n+1}$ would not form a Cauchy sequence in $X$. It is therefore easy to see that the sequences $S_{m}$ converge pointwise and hence in the metric, and that the limit is in $\Sigma(X)$. It is also simple to check that this metric gives rise to the N-topology on $\Sigma(X)$.

Proof of Theorem 4.1. Since $\Sigma$ is a Polish space (in the N-topology) we can use the equivalent definition of analytic sets. Suppose therefore that $\sigma \subset \Sigma$ and that there is a continuous map $f: \mathcal{N} \rightarrow \Sigma$ such that $f(\mathcal{N})=\sigma$. Given a finite sequence $n_{1}, \ldots, n_{k}$ of integers, let $\sigma_{n_{1}, \ldots, n_{k}}$ denote the image under $f$ of the set of sequences in $\mathcal{N}$ starting $n_{1}, \ldots, n_{k}$. Note that, for any $\Delta>0$, $\left(\sigma_{n_{1}, \ldots, n_{k}}\right)_{\Delta}=\bigcup_{n_{k+1}=1}^{\infty}\left(\sigma_{n_{1}, \ldots, n_{k+1}}\right)_{\Delta}$.

Let $0=\Delta_{0}, \Delta_{1}, \Delta_{2}, \ldots$ be a (pointwise) strictly increasing sequence of real sequences less than $\Delta$. For $n \geqslant 0$ let $\Gamma_{n}$ be the sequence $\frac{1}{4}\left(\Delta_{n+1}-\Delta_{n}\right)$ and for $i=1,2,3$ let $\Delta_{n, i}=\Delta_{n}+i \Gamma_{n}$.

Let $\phi$ be a bijection between the set of finite sequences of integers and $\mathbb{N}$. By Lemma 4.11 we can find a subspace $W$ of $X$ such that, for every $\left(n_{1}, \ldots, n_{k}\right)$ and every sequence $A \in \Sigma_{f}(W)$ of length at least $\phi\left(n_{1}, \ldots, n_{k}\right)$, either $\left(\sigma_{n_{1}, \ldots, n_{k}}\right)_{\Delta_{k, 1}} \cap[A ; W]$ is empty or $\left(\sigma_{n_{1}, \ldots, n_{k}}\right)_{\Delta_{k, 2}}$ is large for $[A ; W]$.

For every $\left(n_{1}, \ldots, n_{k}\right)$ and every pair $(A, Z)$ in $W$, define $\rho_{n_{1}, \ldots, n_{k}}[A ; Z]$ to be the set of all sequences $B \in \Sigma_{f}(Z)$ such that there exists $n_{k+1}$ with $\phi\left(n_{1}, \ldots, n_{k+1}\right)$ at most the length of $B$ and $\left(\sigma_{n_{1}, \ldots, n_{k+1}}\right)_{\Delta_{k, 3}}$ large for $[A, B ; Z]$. Let $\Pi_{n_{1}, \ldots, n_{k}}$ be the set of pairs $(A, Z)$ such that if $A$ has length at least $\phi\left(n_{1}, \ldots, n_{k}\right)$ then either

(a) $\left(\sigma_{n_{1}, \ldots, n_{k}}\right)_{\Delta_{k, 1}} \cap[A ; Z]$ is empty

or

(b) $\rho_{n_{1}, \ldots, n_{k}}[A ; Z]$ is strategically large for $Z$.

We shall apply Lemma 2.2 to the sets $\Pi_{n_{1}, \ldots, n_{k}}$. However, unlike with our previous diagonalization arguments, it will not be trivial to verify that the relevant conditions are satisfied. Instead, we use Lemma 4.12. Condition (ii) of Lemma 2.2 is of course not a problem. As for condition (i), let $(A, Z)$ be any pair and suppose that the length of $A$ is at least $\phi\left(n_{1}, \ldots, n_{k}\right)$. If (a) is not true for the pair $(A, Z)$, then by our construction of the subspace $W$ we know that $\left(\sigma_{n_{1}, \ldots, n_{k}}\right)_{\Delta_{k, 2}}$ is large for $[A ; W]$ and hence for $[A ; Z]$. Now

$$
\left(\sigma_{n_{1}, \ldots, n_{k}}\right)_{\Delta_{k, 2}}=\bigcup_{n_{k+1}=1}^{\infty}\left(\sigma_{n_{1}, \ldots, n_{k+1}}\right)_{\Delta_{k, 2}} .
$$

Lemma 4.12 applied to this union with $\Delta=\Gamma_{k}$ implies that there exists a subspace $Z^{\prime} \subset Z$ such that $\rho_{n_{1}, \ldots, n_{k}}\left[A ; Z^{\prime}\right]$ is strategically large for $Z^{\prime}$, which tells us that (b) is true of the pair $\left(A, Z^{\prime}\right)$. This shows that condition (ii) of Lemma 2.2 is indeed satisfied. 
Using that lemma, we can find a subspace $Y \subset W$ such that every pair $(A, Z)$ in $Y$ with $A$ of length at least $\phi\left(n_{1}, \ldots, n_{k}\right)$ belongs to $\left(\Pi_{n_{1}, \ldots, n_{k}}\right)_{\Gamma_{k}}$. Let us define perturbations of the sets $\rho_{n_{1}, \ldots, n_{k}}[A ; Z]$ by defining $\tau_{n_{1}, \ldots, n_{k}}[A ; Z]$ to be the set of all sequences $B \in \Sigma_{f}(Z)$ such that there exists $n_{k+1}$ with $\phi\left(n_{1}, \ldots, n_{k+1}\right)$ at most the length of $B$ and $\left(\sigma_{n_{1}, \ldots, n_{k+1}}\right)_{\Delta_{k+1}}$ large for $[A, B ; Z]$. The statement that $(A, Z)$ belongs to $\left(\Pi_{n_{1}, \ldots, n_{k}}\right)_{\Gamma_{k}}$ implies that either $\left(\sigma_{n_{1}, \ldots, n_{k}}\right)_{\Delta_{k}} \cap[A ; Z]$ is empty or $\tau_{n_{1}, \ldots, n_{k}}[A ; Z]$ is strategically large for $Z$. This is true in particular when $Z=Y$.

We claim now that $\sigma_{\Delta}$ is strategically large for the subspace $Y$, and to prove it we shall describe a suitable strategy for $\mathrm{P}$. The game starts with $\sigma=\sigma_{\Delta_{0}}$ large for $Y$. By our construction of $Y$, the set $\tau$ is strategically large for $Y$. That means that $\mathrm{P}$ can ensure that after some finite number of moves (s)he will reach a sequence $B_{1} \in \Sigma_{f}(Y)$ such that there exists $n_{1}$ with $\phi\left(n_{1}\right)$ at most the length of $B_{1}$ and $\left(\sigma_{n_{1}}\right)_{\Delta_{1}}$ large for $\left[B_{1} ; Y\right]$. In general, suppose that $\mathrm{P}$ has reached a sequence $B_{k} \in \Sigma_{f}(Y)$ such that there exists $\left(n_{1}, \ldots, n_{k}\right)$ with $\phi\left(n_{1}, \ldots, n_{k}\right)$ at most the length of $B_{k}$ and $\left(\sigma_{n_{1}, \ldots, n_{k}}\right)_{\Delta_{k}}$ large for $\left[B_{k} ; Y\right]$. The construction of $Y$ guarantees that $\tau_{n_{1}, \ldots, n_{k}}$ is strategically large for $Y$, which means that $\mathrm{P}$ can play in such a way as to extend $B_{k}$ to a sequence $B_{k+1}$ such that there exists $n_{k+1}$ with $\phi\left(n_{1}, \ldots, n_{k+1}\right)$ at most the length of $B_{k+1}$ and $\left(\sigma_{n_{1}, \ldots, n_{k+1}}\right)_{\Delta_{k+1}}$ large for $\left[B_{k+1} ; Y\right]$.

Let $\mathrm{P}$ play as above and let $B$ be the infinite sequence which has the finite sequences $B_{k}$ as initial segments. That is, $B$ is the sequence produced by $\mathrm{P}$ at the end of the game. It remains to prove that $B \in \sigma_{\Delta}$. We do this by showing that $d\left(B, f\left(n_{1}, n_{2} \ldots\right)\right) \leqslant \Delta$. Write $B=\left(x_{n}\right)_{n=1}^{\infty}$ and $f\left(n_{1}, n_{2}, \ldots\right)=$ $\left(y_{n}\right)_{n=1}^{\infty}$. If our assertion is false then there exists $t$ such that $d\left(x_{t}, y_{t}\right)>\delta_{t}$. By the continuity of $f$, for every sufficiently large $k$ and for every sequence $\left(m_{1}, m_{2}, \ldots\right) \in \mathcal{N}$ with $m_{i}=n_{i}$ for all $i \leqslant k$, if $z=f\left(m_{1}, m_{2}, \ldots\right)$, then $d\left(z_{t}, y_{t}\right)<\delta_{t}-d\left(x_{t}, y_{t}\right)$. For any $r \geqslant \max \left\{t, \phi\left(n_{1}, \ldots, n_{k}\right)\right\}$, we have just proved that $\left(\sigma_{n_{1}, \ldots, n_{k}}\right)_{\Delta} \cap\left[x_{1}, \ldots, x_{r} ; Y\right]$ is empty, which implies that $\left(\sigma_{n_{1}, \ldots, n_{k}}\right)_{\Delta_{k}}$ is not large for $\left[x_{1}, \ldots, x_{r} ; Y\right]$. Choosing $k$ such that $\phi\left(n_{1}, \ldots, n_{k}\right)>t$, we find that there does not exist $r \geqslant \phi\left(n_{1}, \ldots, n_{k}\right)$ such that $\left(\sigma_{n_{1}, \ldots, n_{k}}\right)_{\Delta_{k}}$ is large for $\left[B_{k} ; Y\right]$, which is a contradiction.

\section{Spaces where the result can be strengthened}

We show first how to strengthen Theorem 4.1 to a genuine Ramsey result when the Banach space given at the beginning is $c_{0}$ and the block bases are normalized. The big difference between $c_{0}$ and other spaces is the following theorem [G5]. 
THEOREM 6.1. Let $A_{1} \cup \ldots \cup A_{r}$ be a partition of the unit sphere of $c_{0}$ and let $\varepsilon>0$. Then there exists an infinite-dimensional block subspace $Y$ of $c_{0}$ and some $j \leqslant r$ such that every point in the unit sphere of $Y$ is within $\varepsilon$ of some point of $A_{j}$.

Define a subset $A$ of the unit sphere of $c_{0}$ to be large if it has a nonempty intersection with every block subspace of $c_{0}$. (The word asymptotic is more standard in Banach space theory, but less in keeping with the terminology of this paper and other papers in Ramsey theory.) An equivalent formulation of Theorem 1 is that if $A$ is a large subset of $c_{0}$ and $\varepsilon>0$ then there exists an infinite-dimensional subspace $Y$ of $c_{0}$ such that every point of $Y$ lies within $\varepsilon$ of some point of $A$.

We shall now prove something similar for analytic sets of normalized block bases of $c_{0}$ (or equivalently, of block subspaces of $c_{0}$ ). Rather than giving full details, which are very similar to the proof of Theorem 4.1, we merely indicate the points at which that argument must be changed. Let $\Sigma_{1}(X)$ denote the set of all normalized block bases of $X$ and let us now interpret notation such as $[X]$ to refer to normalized block bases. (Alternatively, one could consider sets $\sigma \subset \Sigma(X)$ such that $\left(\left(x_{n}, \lambda_{n}\right)\right)_{n=1}^{\infty} \in \sigma$ implies that $\left(\left(x_{n}, \mu_{n}\right)\right)_{n=1}^{\infty}$ for all sequences $\left(\mu_{n}\right)_{n=1}^{\infty}$ of numbers in the interval $[0,1]$.) Let us define a subset $\sigma$ of $\Sigma_{1}\left(c_{0}\right)$ to be Ramsey if for every $\Delta>0$ there is a block subspace $Y$ of $c_{0}$ such that either $\sigma \cap[Y]$ is empty or $[Y] \subset \sigma_{\Delta}$.

\section{Lemma 6.2. $\mathrm{N}$-closed subsets of $\Sigma_{1}\left(c_{0}\right)$ are Ramsey.}

Proof. Corollary 4.7 implies that N-closed subsets of $\Sigma\left(c_{0}\right)$ are weakly Ramsey. Let $\sigma$ be N-closed and large for $c_{0}$, let $\Delta>0$ and let $X$ be a block subspace of $c_{0}$ such that $\sigma_{\Delta / 2}$ is strategically large for $X$. Let $\Delta / 2=\Delta_{0}<$ $\Delta_{0}^{+}<\Delta_{1}^{-}<\Delta_{1}<\Delta_{1}^{+}<\Delta_{2}^{-}<\Delta_{2}<\ldots$ be a strictly increasing sequence of sequences bounded above by $\Delta$. For each $k$ let $\Pi_{k}$ be the set of pairs $(A, Z)$ such that $A$ has length $k$ and either

or

(a) for every $z \in Z, \sigma_{\Delta_{k}^{+}}$is not strategically large for $[(A, z) ; X]$

(b) for every $z \in Z, \sigma_{\Delta_{k+1}^{-}}$is strategically large for $[(A, z) ; X]$.

Clearly, if $(A, Z) \in \Pi_{k}$ and $Z^{\prime}$ is a subspace of $Z$ then $\left(A, Z^{\prime}\right) \in \Pi_{k}$. We now verify condition (i) of Lemma 2.2. Let $(A, Z)$ be a pair such that (a) is not true for any subspace of $Z$. This says that the set of $z \in Z$ for which $\sigma_{\Delta_{k}^{+}}$is strategically large for $[(A, z) ; X]$ is large for $Z$. Hence, by Theorem 6.1 there is a subspace $Z^{\prime} \subset Z$ such that for every $z \in Z^{\prime}$ the set $\sigma_{\Delta_{k+1}^{-}}$is strategically large for $[(A, z) ; X]$. (Note that we did not use the full strength of the fact that $\Delta_{k}^{+}<\Delta_{k+1}^{-}$. The strict inequality was important only for the $k^{\text {th }}$ terms of these sequences.) 
We may therefore apply Corollary 2.4, which, for a suitable choice of perturbations, gives us a subspace $Y$ of $X$ such that, for every finite normalized block basis $A$ in $Y$, either

(c) for every $z \in Y, \sigma_{\Delta_{k}}$ is not strategically large for $[(A, z) ; X]$

or

(d) for every $z \in Y, \sigma_{\Delta_{k+1}}$ is strategically large for $[(A, z) ; X]$.

We claim now that $[Y] \subset \sigma_{\Delta}$. The proof is by induction. We start with the statement that $\sigma_{\Delta_{0}}$ is strategically large for $[X]$. It follows that there exists $z \in S(Y)$ such that $\sigma_{\Delta_{0}}$ is strategically large for $X$ (or else $\mathrm{S}$ would have a winning strategy for the game $\sigma_{\Delta_{0}}[X]$ by starting with the subspace $Y)$. By construction of $[Y]$, for every $z_{1} \in S(Y)$ the set $\sigma_{\Delta_{1}}$ is strategically large for $\left[z_{1} ; X\right]$. By a similar argument, for any fixed $z_{1}$ we then have that for every $z_{2} \in S(Y)$ the set $\sigma_{\Delta_{2}}$ is strategically large for $\left[z_{1}, z_{2} ; X\right]$. Continuing in this way we find that for any finite normalized block basis $\left(z_{1}, \ldots, z_{k}\right)$ in $Y$, $\sigma_{\Delta_{k}}$ is strategically large for $\left[z_{1}, \ldots, z_{k} ; X\right]$. It follows that $\left(z_{1}, \ldots, z_{k}\right)$ can be extended to a sequence in $\sigma_{\Delta}$. By Lemma 4.6, $\sigma_{\Delta}$ is N-closed, and therefore D-closed. It follows that every sequence $\left(z_{n}\right)_{n=1}^{\infty} \in \Sigma_{1}(Y)$ belongs to $\sigma_{\Delta}$.

Corollary 6.3. N-open subsets of $\Sigma_{1}\left(c_{0}\right)$ are Ramsey.

Proof. Let $\sigma$ be an N-open subset of $\Sigma_{1}\left(c_{0}\right)$ and let $\tau$ be the complement of $\sigma_{\Delta}$. It is not hard to show that $\sigma_{\Delta}$ is N-open, so $\tau$ is N-closed. If no subpace $Y$ can be found such that $[Y] \subset \sigma_{\Delta}$, then $\tau$ is large for $c_{0}$. Then, by Lemma 6.2 , there is a subspace $Y$ such that $[Y] \subset \tau_{\Delta}$. However, $\tau_{\Delta} \cap \sigma$ is empty, so $\sigma \cap[Y]$ is empty.

Given a subspace $Y$ of $c_{0}$ and a collection $\rho$ of finite sequences, let us say that $\rho$ is very large for $Y$ if every sequence in $\Sigma_{1}(Y)$ has an initial segment in $\rho$.

Lemma 6.4. Let $\sigma \subset \Sigma_{1}\left(c_{0}\right)$ be large, let $\sigma=\bigcup_{n=1}^{\infty} \sigma_{n}$, let $\Delta>0$ be a real sequence and let $\psi$ be a function from $\mathbb{N}$ to $\mathbb{N}$. For any subspace $Y \subset X$ let $\rho(Y)$ be the set of finite normalized block bases $B$ in $Y$ such that there exists $n$ with $\max \{n, \psi(n)\}$ at most the length of $B$ and $\left(\sigma_{n}\right)_{\Delta}$ large for $[B ; Y]$. Then there exists a subspace $Y \subset X$ such that $\rho(Y)$ is very large for $Y$.

Proof. For each $Y$, let $\tau(Y)$ be defined in the same way as $\rho(Y)$ but with $\Delta$ replaced by $\Delta / 2$. Lemma 4.12 implies that there is a subspace $W$ such that $\tau(W)$ is strategically large for $W$. In particular, $\tau(W)$ is large for $W$. Hence, by Corollary 6.3, there is a subspace $Y \subset W$ such that $[Y] \subset \tau(W)_{\Delta / 2}$. The result now follows from the fact that $\tau(W)_{\Delta / 2} \subset \rho(W)$ and that $\rho(Y) \subset[Y] \cap \rho(W)$. 
TheOREM 6.5. Let $\sigma$ be an $\mathrm{N}$-analytic subset of $\Sigma_{1}\left(c_{0}\right)$ and let $\Delta>0$. Then there is a subspace $X \subset c_{0}$ such that either $[X] \cap \sigma=\emptyset$ or $[X] \subset \sigma_{\Delta}$.

Proof (Sketch). The proof is very similar to that of Theorem 4.1. The main changes are that the phrase "strategically large" should be replaced by "very large" and that instead of Lemma 4.12 one should use Lemma 6.4. In the penultimate paragraph of the proof, instead of considering a game between $\mathrm{P}$ and $\mathrm{S}$, one considers an arbitrary sequence $B$ in $\Sigma_{1}(Y)$. The construction of $Y$ now guarantees that this sequence has an increasing collection of initial segments $B_{1}, B_{2}, \ldots$ such that for some sequence $n_{1}, n_{2}, \ldots$ of positive integers, we have for every $k$ that $\phi\left(n_{1}, \ldots, n_{k}\right)$ is at most the length of $B_{k}$ and $\left(\sigma_{n_{1}, \ldots, n_{k}}\right)_{\Delta_{k}}$ is large for $\left[B_{k} ; Y\right]$. The proof that the distance between $B$ and $f\left(n_{1}, n_{2}, \ldots\right)$ is at most $\Delta$ is the same as in the proof of Theorem 4.1.

The above result also applies, for trivial reasons, to spaces containing $c_{0}$. It follows from results of Milman [Mi] and Odell and Schlumprecht [OS] that these are the only spaces for which this stronger Ramsey result is true. The result can in fact be strengthened further; it is basically symmetrical and so an Ellentuck-type theorem holds.

Going to the opposite extreme, let us consider the "worst" spaces and return to nonnormalized sequences. Recall that a space $X$ (which we assume for convenience has a basis here) is hereditarily indecomposable if it has one of the following equivalent properties:

(i) $X$ has no subspace that can be decomposed as a topological direct sum $Y \oplus Z$ with $Y$ and $Z$ infinite-dimensional;

(ii) for any pair of infinite-dimensional subspaces $Y$ and $Z$ of $X$ and any $\varepsilon>0$ there exist $y \in Y$ and $z \in Z$ such that $\varepsilon\|y+z\|>\|y-z\|$;

(iii) for any pair of infinite-dimensional block subspaces $Y$ and $Z$ of $X$ and any positive real sequence $\Delta$ there are block subspaces $Y^{\prime} \subset Y$ and $Z^{\prime} \subset Z$ such that $d\left(Y^{\prime}, Z^{\prime}\right) \leqslant \Delta$.

The third property above, which we shall use, states that up to small perturbations the subspaces of $X$ form a filter. The existence of such spaces is proved in [GM1]. The observation we make here is that if $X$ is hereditarily indecomposable and $\sigma \subset \Sigma(X)$ is a set such that, for some subspace $Y \subset X$, $\mathrm{P}$ has a winning strategy for the game $\sigma[Y]$, then for any $\Delta>0 \mathrm{P}$ has a winning strategy for the game $\sigma_{\Delta}[X]$. Indeed, let $X_{1}, X_{2}, \ldots$ be any sequence of (infinite-dimensional block) subspaces of $X$. Using property (iii) with the perturbations sufficiently small, we can find a sequence of subspaces $Y_{1}, Y_{2}, \ldots$ such that for every $n \in \mathbb{N}$ and every $y \in Y_{n}$ of norm 1 there exists $x \in X_{n}$ of 
norm 1 such that $\|y-x\|<\delta_{n}$. If $\mathrm{P}$ plays a (fixed) winning strategy against the moves $Y_{1}, Y_{2}, \ldots$ from $\mathrm{S}$, then $\mathrm{P}$ has produced a sequence in $\sigma_{\Delta}$. Thus we have the following result.

TheOREm 6.6. Let $X$ be a hereditarily indecomposable space, let $\Delta>0$ and let $\sigma \subset \Sigma(X)$ be $\mathrm{N}$-analytic. Then either there is a subspace $Y$ such that $\sigma \cap[Y]=\emptyset$ or there is a winning strategy for $P$ for the game $\sigma_{\Delta}[X]$.

\section{Applications}

Our aim in this section is to obtain the beginnings of a classification of separable infinite-dimensional Banach spaces. We do not mean by this a complete description of all such spaces up to isomorphism, since such a task is clearly hopeless. Rather, we wish to find classes of Banach spaces with the following properties:

(a) If $X$ is in one of the classes, then so is every subspace of $X$.

(b) Every space has a subspace in one of the classes.

(c) The classes are very obviously disjoint.

(d) Knowing that a space belongs to a particular class gives a lot of information about the structure of the space and what operators can be defined on it.

Properties (c) and (d) above are of course related. We begin with the comment that it is slightly easier to deduce Theorem 1.4 from Theorem 4.1 than from Theorem 2.1, because the extra diagonalization (see the end of $\S 3$ ) can be avoided. One defines $\sigma$ to be the set of all infinite sequences $\left(x_{n}, \lambda_{n}\right)_{n=1}^{\infty}$ such that for every positive integer $m$ there exist $r$ and $s$ such that the sequence $\left(\lambda_{r} x_{r}, \lambda_{r+1} x_{r+1}, \ldots, \lambda_{s} x_{s}\right)$ is $m$-conditional. Lemma 1.6 implies that $X$ contains no unconditional basic sequence if and only if $\sigma$ is large. It is easy to check that $\sigma$ is a $\mathrm{G}_{\delta}$-set. So if $X$ contains no unconditional basic sequence and $\Delta>0$, then we can find a subspace $Y$ of $X$ such that $\sigma_{\Delta}$ is strategically large for $Y$. For sufficiently small $\Delta$, this shows, as in the proof of Corollary 3.2, that $Y$ satisfies condition (ii) of Lemma 1.7, and is therefore hereditarily indecomposable.

In [GM1] it was shown that every operator on a (complex) hereditarily indecomposable space is a strictly singular perturbation of a multiple of the identity. This result was strengthened by Ferenczi [F2], who proved that every operator from a subspace of a hereditarily indecomposable space into the space itself is a strictly singular perturbation of the inclusion map. We have therefore 
found two classes satisfying properties (a) to (d). However, property (d) is a matter of degree. It is not clear whether one would want to classify the hereditarily indecomposable spaces any further (at least from the point of view of understanding the operators defined on them) but there is a wide variety of spaces with an unconditional basis differing in important ways. At one extreme there are the $\ell_{p}$ spaces, the ones with most symmetry, and at the other is a space constructed in [G4] which is not isomorphic to any proper subspace of itself. In fact, it is shown in [GM2] that every operator on the space is a strictly singular perturbation of a diagonal map. (It is not known whether every operator from a subspace to the whole space is a strictly singular perturbation of the restriction of a diagonal map.) Since the diagonal maps with bounded entries down the diagonal are trivially continuous, such a space can be regarded as the "worst" type of space with an unconditional basis, just as a hereditarily indecomposable space is the "worst" type of general space.

It is natural to ask, therefore, what can be said about a space with an unconditional basis if it does not contain the "worst" kind of subspace. Is there some result stating that such a space must have a "nice" subspace? We shall give a result of this type, but we need some definitions first.

Recall first that an infinite-dimensional Banach space $X$ is said to be minimal if every infinite-dimensional subspace of $X$ has a further subspace isomorphic to $X$. This term was coined by Rosenthal, who conjectured that every infinite-dimensional space has a minimal subspace. However, it was shown by Casazza and Odell [CO] that Tsirelson's space gives a counterexample. Of course, a hereditarily indecomposable space gives a much stronger counterexample, but for this very reason Tsirelson's space is a more interesting one. It has in particular a property which we shall now define and discuss in some detail. Recall that two spaces $X$ and $Y$ are said to be totally incomparable if no infinite-dimensional subspace of $X$ is isomorphic to a subspace of $Y$. Let us say that a space $X$ is quasi-minimal if it does not contain a pair of totally incomparable subspaces. The reason for this terminology is given by the next simple lemma. If $Y$ and $Z$ are two spaces, we write $Y \leqslant Z$ to mean $Y$ embeds into $Z$.

Lemma 7.1. A space $X$ is quasi-minimal if and only if there is a collection $\mathcal{S}$ of subspaces of $X$ with the following properties:

(i) The set $\mathcal{S}$ is partially ordered by $\leqslant$.

(ii) If $Y, Z \in \mathcal{S}$ then there exists $W \in \mathcal{S}$ such that $Y \geqslant W$ and $Z \geqslant W$.

(iii) Every subspace of $X$ has a subspace isomorphic to a subspace in $\mathcal{S}$. 
Proof. Write $Y \sim Z$ if $Y \leqslant Z$ and $Z \leqslant Y$. Then $\sim$ is an equivalence relation. If $\mathcal{S}$ consists of a representative from each equivalence class then it clearly has the properties stated. The converse is obvious from properties (ii) and (iii).

Remarks. The important property is of course (ii). If a quasi-minimal space $X$ has a minimal subspace $Y$ then $Y$ embeds into every subspace of $X$. (Under these circumstances $X$ is said to be $Y$-saturated.) For if $Z$ were a subspace into which $Y$ did not embed then $Y$ would embed into every subspace of $Y$ and no subspace of $Z$, so $Y$ and $Z$ would be totally incomparable. It follows that $X$ has a minimal subspace if and only if $\mathcal{S}$ has cardinality one for every choice of $\mathcal{S}$. If $\mathcal{S}$ has cardinality greater than one, in which case we shall say that $X$ is strictly quasi-minimal, then $\mathcal{S}$ is uncountable. To see this, suppose $\mathcal{S}$ is countably infinite (the finite case is easier). We can pick a chain $Y_{1}>Y_{2}>\ldots$ of subspaces in $\mathcal{S}$ such that every $Y \in \mathcal{S}$ satisfies $Y>Y_{n}$ for some $n$. Now let $z_{1}<z_{2}<\ldots$ with $z_{n} \in Y_{n}$ and let $Z$ be the subspace spanned by $z_{1}, z_{3}, z_{5}, \ldots$ Then some $Y_{n}$ embeds into $Z$. However, $Z$ is isomorphic to the subspace spanned by $z_{n+1}, z_{n+2}, \ldots, z_{2 n}, z_{2 n+1}, z_{2 n+3}, z_{2 n+5}, \ldots$ which is a subspace of $Y_{n+1}$, contradicting the fact that $Y_{n}>Y_{n+1}$. Finally, observe that a hereditarily indecomposable space is quasi-minimal for trivial reasons. The definition will therefore only interest us in the context of spaces with an unconditional basis.

We say that two block subspaces $Y$ and $Z$ of a space $X$ are disjointly supported if the support of every $y \in Y$ is disjoint from the support of every $z \in Z$. We can now state and prove a dichotomy for spaces with an unconditional basis. Later we shall strengthen it a little.

TheOREM 7.2. Let $X$ be a Banach space with an unconditional basis. Then either $X$ has a quasi-minimal subspace or $X$ has a subspace $Y$ such that no two disjointly supported subspaces of $Y$ are isomorphic (and hence any two disjointly supported subspaces are totally incomparable).

Although it is possible to prove Theorem 7.2 in one go using Theorem 4.1 , it is perhaps of interest to show that the full strength of Theorem 4.1 is by no means necessary. Instead we shall use Corollary 4.7 (that N-closed sets are weakly Ramsey) and a diagonalization. In order to do this, we must, as with Theorem 1.4, introduce some more quantitative definitions. Given two block subspaces $Y, Z$ of a Banach space $X$, we shall say that they are $C$-block isomorphic if the linear map from $Y$ to $Z$ taking the normalized block basis of $Y$ to the normalized block basis of $Z$ is a $C$-isomorphism. We shall say that a space $X$ is $C$-quasi-minimal if any two block subspaces $Y, Z$ have 
further subspaces $V, W$ which are $C$-block isomorphic. In the next lemma, when $A$ and $B$ are finite subsets of $\mathbb{N}$, we write $A<B$ for the statement that $\max A<\min B$.

LEMma 7.3. Let $X$ be a Banach space with an unconditional basis and let $C \geqslant 1$ and $\varepsilon>0$. Then either $X$ has a $(C+\varepsilon)$-quasi-minimal subspace or $X$ has a subspace $V$ such that no two disjointly supported block subspaces of $V$ are C-block isomorphic.

Proof. For convenience we shall assume that $X$ has the property that $\|x+y\|>\|x\|$ whenever $x<y$ are nonzero vectors in $X$. Standard arguments tell us that every Banach space (with a given monotone basis) is almost isometric to a space with this property. Suppose that every block subspace $W$ of $X$ contains a pair of disjointly supported $C$-block-isomorphic subspaces $Y, Z$. By passing to subsequences we may assume that $Y$ and $Z$ are generated by normalized block bases $\left(y_{n}\right)_{n=1}^{\infty}$ and $\left(z_{n}\right)_{n=1}^{\infty}$ such that $y_{i}<z_{i+1}$ and $z_{i}<y_{i+1}$ for every $i \geqslant 1$. We know that for every $i \geqslant 1$ the vectors $y_{i}$ and $z_{i}$ are disjointly supported linear combinations of the vectors in the block basis of $W$. Therefore, we can find a sequence $w_{1}<w_{2}<\ldots$ of nonzero vectors in the unit ball of $W$ and a partition $A_{1}<A_{2}<\ldots$ of $\mathbb{N}$ such that, if we let $y_{i}$ be the sum of all $w_{j}$ such that $j \in A_{i}$ is odd, and $z_{i}$ be the sum of all $w_{j}$ such that $j \in A_{i}$ is even, then the sequences $y_{1}, y_{2}, \ldots$ and $z_{1}, z_{2}, \ldots$ are $C$-equivalent normalized block bases. Let $\sigma$ be the set of sequences $\left(w_{n}\right)_{n=1}^{\infty}$ for which such a partition of $\mathbb{N}$ exists. (Of course, strictly speaking we should replace each $w_{i}$ by the pair $\left(w_{i} /\left\|w_{i}\right\|,\left\|w_{i}\right\|\right)$.)

Notice that, given our sequence $w_{1}, w_{2}, \ldots$, the sets $A_{i}$, and hence the vectors $y_{i}$ and $z_{i}$, are uniquely determined. For example, $A_{1}=\{1, \ldots, n\}$, where $n$ is the unique positive integer such that the sum of all $w_{j}$ with $j \leqslant n$ odd has norm 1 and so does the sum of all $w_{j}$ with $j \leqslant n$ even. Once $A_{1}$ has been determined, the uniqueness of $A_{2}$ follows similarly, and so on. The sequences $y_{1}, y_{2}, \ldots$ and $z_{1}, z_{2}, \ldots$ are $C$-equivalent if and only if all their initial segments are $C$-equivalent. By the uniqueness of the sets $A_{i}$, this is a property of initial segments of the sequence $w_{1}, w_{2}, \ldots$ Moreover, if $w_{1}, w_{2}, \ldots$ is a sequence not in $\sigma$, either because we cannot find the sets $A_{i}$ making the vectors $y_{i}$ and $z_{i}$ normalized, or because for some $n$ the sequences $y_{1}, \ldots, y_{n}$ and $z_{1}, \ldots, z_{n}$ are not $C$-equivalent, then a sufficiently small perturbation of $w_{1}, w_{2}, \ldots$ will also not be in $\sigma$. This shows that $\sigma$ is N-closed.

Since $\sigma$ is closed and large for $X$, Corollary 4.7 allows us, for any $\Delta>0$, to pass to a subspace $V \subset X$ such that $\sigma_{\Delta}$ is strategically large for $Y$. It is not hard to show that if $\Delta$ is sufficiently small, then for any sequence $\left(w_{n}\right)_{n=1}^{\infty} \in \sigma_{\Delta}$ there are normalized sequences $\left(y_{n}\right)_{n=1}^{\infty}$ and $\left(z_{n}\right)_{n=1}^{\infty}$ which are $(C+\varepsilon)$-equivalent such that the $y_{i}$ are generated by $w_{1}, w_{3}, \ldots$ and the $z_{i}$ are 
generated by $w_{2}, w_{4}, \ldots$ Since $\sigma_{\Delta}$ is strategically large for $V$, if $\mathrm{S}$ chooses any two subspaces $Y, Z$ of $V$ and plays the strategy $Y, Z, Y, Z, \ldots, \mathrm{P}$ can find a sequence $\left(w_{n}\right)_{n=1}^{\infty} \in \sigma_{\Delta}$ with $w_{i} \in Y$ for $i$ odd and $w_{i} \in Z$ for $i$ even. Choosing sequences $\left(y_{n}\right)_{n=1}^{\infty}$ and $\left(z_{n}\right)_{n=1}^{\infty}$ as above, we find block subspaces of $Y$ and $Z$ which are $(C+\varepsilon)$-block isomorphic.

Proof of Theorem 7.2. Suppose that $X$ has no quasi-minimal subspace. Then obviously $X$ has no $m$-quasi-minimal subspace for any positive integer $m$. Therefore by Lemma 7.3 we can find a nested sequence of subspaces $W_{1} \supset$ $W_{2} \supset \ldots$ such that no two disjointly supported block subspaces of $Y_{m}$ are $(m-1)$-block-isomorphic. Let $w_{1}<w_{2}<\ldots$ be a normalized block basis with $w_{m} \in W_{m}$ for every $m$ and let $W$ be the subspace generated by $w_{1}, w_{2}, \ldots$ We claim that no two disjointly supported subspaces of $W$ are isomorphic.

To see this, suppose that $Y$ and $Z$ are two disjointly supported block subspaces of $W$. If they are isomorphic, then by standard arguments there exist equivalent block bases $\left(y_{n}\right)_{n=1}^{\infty}$ and $\left(z_{n}\right)_{n=1}^{\infty}$ with every $y_{i}$ in $Y$ and every $z_{i}$ in $Z$ (but not necessarily generating the whole of $Y$ and $Z$ ). They must be $C$ equivalent for some $C$, so let us choose a positive integer $m \geqslant C+2$, and suppose (by removing the beginnings) that every $y_{i}$ and every $z_{i}$ lies in $W_{m}$. We are not quite finished because the $y_{i}$ and $z_{i}$ are not necessarily normalized. However, the ratio $\left\|y_{i}\right\| /\left\|z_{i}\right\|$ is bounded and bounded away from zero. Therefore, we can drop to subsequences such that this ratio converges very quickly to a nonzero limit, and then a small perturbation gives us normalized block bases which are $(C+1)$-equivalent. This then contradicts our choice of $W_{m}$.

It is clear that the dichotomy above is genuinely a dichotomy. If a space even contains two disjointly supported totally incomparable subspaces then it is not quasi-minimal. As we mentioned above, it is possible to prove Theorem 7.2 more directly. If we let $\sigma_{m}$ be the set $\sigma$ defined in the proof of Lemma 7.3 when $C=m$ and let $\sigma=\bigcup_{m=1}^{\infty}$, then $\sigma$ is an $\mathrm{F}_{\sigma}$-set. If every subspace contains two isomorphic disjointly supported further subspaces, then the argument in the deduction of Theorem 7.2 from Lemma 7.3 shows that $\sigma$ is large. Applying Theorem 4.1, we can find a subspace $W$ where $\sigma_{\Delta}$ is strategically large which proves, as above, that $W$ is quasi-minimal.

Notice that the proof of Theorem 7.2 actually gave us something more: every Banach space $X$ has a subspace $Y$ such that either $Y$ is $C$-quasi-minimal for some $C$ or $Y$ does not contain two disjointly supported isomorphic subspaces. In particular, every quasi-minimal space has a subspace which is $C$-quasi-minimal for some $C$.

This last result is not unlike a consequence of our solution to Banach's problem on homogeneous spaces. It follows from the fact that a homogeneous space $X$ must be a Hilbert space that there exists a constant $C$ such 
that all subspaces of $X$ are $C$-isomorphic. (One might call such a space $C$-homogeneous.) However, no direct proof of this fact is known.

We now give some more information about the "bad" spaces in Theorem 7.2. First, we make a simple observation using a result of Casazza [C]. (The proof can also be found in [G4].)

LEMma 7.4. Let $X$ be a space with a basis such that, for any block basis $y_{1}, z_{1}, y_{2}, z_{2}, \ldots$ the block bases $y_{1}, y_{2}, \ldots$ and $z_{1}, z_{2}, \ldots$ are not equivalent. Then $X$ is isomorphic to no proper subspace of itself (and hence the same is true in any subspace of $X)$.

Corollary 7.5. Let $X$ be a space with an unconditional basis such that no two disjointly supported subspaces are isomorphic. Then no subspace of $X$ is isomorphic to a further proper subspace.

Proof. Clearly such a space satisfies the above criterion of Casazza.

We shall now strengthen Corollary 7.5 considerably. In the proof below, we identify a set $A \subset \mathbb{N}$ with the obvious projection on $X$ associated with $A$. The result basically states that two block subspaces of a space $X$ satisfying the conditions of Corollary 7.5 are only isomorphic if they are isomorphic for trivial reasons.

Lemma 7.6. Let $X$ be a space with an unconditional basis such that no two disjointly supported subspaces are isomorphic, and let $Y$ and $Z$ be subspaces of $X$ generated by equivalent block bases. Let $T: Y \rightarrow Z$ be the corresponding isomorphism. Then there is an invertible diagonal operator $D: X \rightarrow X$ such that $T-\left.D\right|_{Y}$ and $T^{-1}-\left.D^{-1}\right|_{Z}$ are strictly singular.

Proof. Let $\left(y_{n}\right)_{n=1}^{\infty}$ be a normalized block basis generating $Y$ and let $z_{n}=$ $T y_{n}$ for every $n$. Without loss of generality the basis of $X$ is 1-unconditional. Let $c, C$ be constants such that $c\|y\| \leqslant\|T y\| \leqslant C\|y\|$ for every $y \in Y$. For each $n$ let $J_{n}, K_{n}$ and $L_{n}$ be respectively the sets of integers $m$ such that $0<$ $2 C\left|y_{n m}\right| \leqslant\left|z_{n m}\right|, 2 C\left|y_{n m}\right|>\left|z_{n m}\right|>(c / 2)\left|y_{n m}\right|>0$ and $(c / 2)\left|y_{n m}\right| \geqslant\left|z_{n m}\right|$. Let $J, K$ and $L$ be the unions of the $J_{n}, K_{n}$ and $L_{n}$. Notice that these sets are disjoint.

Now let $D^{\prime}$ be the unique diagonal operator on $K X$ sending $K y_{n}$ to $K z_{n}$ for every $n$. Extend $D^{\prime}$ to an invertible diagonal operator $D$ on the whole of $X$ by mapping $x \in(1-K) X$ to $\lambda x$, where $\lambda$ is a constant between $c$ and $C$. We claim that $D$ has the property required.

We show first that the map $U$ defined on $K Y$ by $K y_{n} \mapsto(J+L) y_{n}$ is continuous. If this were not so, then since $\left(J y_{n}\right)_{n=1}^{\infty}$ and $\left(L y_{n}\right)_{n=1}^{\infty}$ are not equivalent we would be able to find $y \in Y$ such that either $\|J y\| \geqslant 2\|(K+L) y\|$ or $\|L y\|>(4 C / c)\|(J+K) y\|$. Now since the basis of $X$ is 1 -unconditional and 
$\left(z_{n}\right)_{n=1}^{\infty}$ is a block basis, we have in the first case

$$
\begin{aligned}
\|T y\| & \geqslant\|T J y\|-\|T(K+L) y\|>2 C\|J y\|-C\|(K+L) y\| \\
& \geqslant C(\|J y\|+\|(K+L) y\|) \geqslant C\|y\|
\end{aligned}
$$

contradicting the fact that $\|T\| \leqslant C$. Similarly, in the second case, we have

$$
\begin{aligned}
\|T y\| & \leqslant\|T L y\|+\|T(J+K) y\| \\
& \leqslant(c / 2)\|L y\|+C\|(J+K) y\|<(3 c / 4)\|L y\| \leqslant c\|y\|
\end{aligned}
$$

contradicting the fact that $\left\|T^{-1}\right\| \leqslant c^{-1}$. Since $J, K$ and $L$ are disjoint, we also know that $U$ cannot be an isomorphism on any subspace of $K Y$, so it is strictly singular.

It follows that the sequences $\left(y_{n}\right)_{n=1}^{\infty}$ and $\left(K y_{n}\right)_{n=1}^{\infty}$ are equivalent. Thus the map $V$ defined on $K Y$ by $V y_{n}=(1-K) z_{n}$ is continuous and therefore also strictly singular. But $T=(D+V) K=D-D(J+L)+V K$ so $T-D$ is strictly singular as claimed. The argument for $T^{-1}$ is similar.

Putting all these results together, we get the following theorem.

Theorem 7.7. Let $X$ be an infinite-dimensional Banach space. Then $X$ has a subspace $Y$ with one of the following properties, which are mutually exclusive and all possible.

(1) $Y$ is hereditarily indecomposable, and therefore (by [F2]) every operator from a subspace $Z$ of $Y$ into $Y$ is a strictly singular perturbation of a multiple of the inclusion map.

(2) $Y$ has an unconditional basis and every isomorphism between block subspaces $W$ and $Z$ of $Y$ is a strictly singular perturbation of the restriction of some invertible diagonal operator on $Y$.

(3) Y has an unconditional basis and is strictly quasi-minimal.

(4) $Y$ has an unconditional basis and is minimal.

How might Theorem 7.7 be extended? The obvious class to look at is (3). Notice that it is not at all clear that a strictly quasi-minimal space need be isomorphic to a proper subspace. In fact, it is almost certainly not even true. Indeed, a suggested counterexample, with a very sketchy argument about why it was a counterexample, was given in [G3], but so far nobody, the author included, has checked whether the details can be filled in. If such a space exists, it is likely to be the "worst" quasi-minimal space in the sense that the only operators on the space and its subspaces are essentially those guaranteed 
to exist by quasi-minimality. One can then ask what there is to say about a space not containing one of these "worst" subspaces and hope to divide (3) further.

The ultimate extension of Theorem 7.7 would be a sequence of possible and mutually exclusive properties (1) to (k) such that, for any Banach space $X$, there would exist $j$ and a subspace $Y \subset X$ such that $Y$ was the "worst" sort of space satisfying property (j) in the sense that the only operators on $Y$ were those guaranteed by property $(\mathrm{j})$, and such that property $(\mathrm{k})$ was that of being isomorphic to some $\ell_{p}$. Tsirelson's space ought to be an example of a typical space having a property a little stronger than (3) but not as strong as (4). Such a result would in a sense be a complete theory of the structure of operators on subspaces of Banach spaces, as it would show that every space had a subspace that was somehow "generic" of a certain kind, and therefore could be described in some detail.

Unfortunately, it is not altogether obvious what the next step is. Suppose $X$ is strictly quasi-minimal with an unconditional basis and suppose that for every subspace $Y$ of $X$ there are isomorphic subspaces $W, Z$ of $Y$ with $W$ properly contained in $Z$. Then no subspace of $X$ satisfies the criterion of Casazza (Lemma 7.4) so in some further subspace there is a winning strategy for P for producing sequences $\left(x_{n}\right)_{n=1}^{\infty}$ such that the sequence of odd-numbered vectors is equivalent to the sequence of even-numbered ones. This, although a stronger property than quasi-minimality, is rather artificial, and it is not clear what of interest can be deduced from it. We can also find a winning strategy in some subspace for sequences $\left(x_{n}\right)_{n=1}^{\infty}$ such that there is a partition of $\mathbb{N}$ into sets $A_{1}, A_{2}, \ldots$ with $\max \left(A_{i}\right)<\min \left(A_{i+1}\right)$ with $y_{i}=\sum_{j \in A_{i}} x_{j}$ of norm 1 for every $i$ and with $\left(x_{i} /\left\|x_{i}\right\|\right)_{i=1}^{\infty}$ and $\left(y_{n}\right)_{n=1}^{\infty}$ equivalent block bases. Again, it is not clear how to use this fact.

It may well be that there is no tidy dichotomy connected with isomorphisms to proper subspaces. If the following conjecture is true, then it would certainly place a limit on any dichotomy that one might wish to prove.

ConjeCture 7.8. There exists a Banach space $X$ such that every subspace $Y$ of $X$ has further subspaces $Z$ and $W$ such that $Z$ is isomorphic to no proper subspace of itself and $W$ is isomorphic to its hyperplanes.

The difficulty in proving this conjecture is that the known techniques for finding spaces that are not isomorphic to any proper subspace all produce examples such that all subspaces have the same property. A new idea is needed for ruling out operators on the whole space without doing the same for subspaces.

It might be a good idea to focus on the following rather general question. Recall the definitions connected with Lemma 7.1, that $Y \leqslant X$ if $Y$ is isomorphic to a subspace of $X$ and $Y \sim X$ if $Y \leqslant X$ and $X \leqslant Y$. 
Problem 7.9. Given a Banach space $X$, let $\mathcal{P}(X)$ be the set of all equivalence classes of subspaces of $X$, partially ordered as above. For which posets $P$ does there exist a Banach space $X$ such that every subspace $Y$ of $X$ contains a further subspace $Z$ with $\mathcal{P}(Z)=P$ ?

Characterizing all such posets might be rather difficult, if it involved constructing exotic Banach spaces. However, even a strong necessary condition, perhaps proved using the methods of this paper, would be interesting.

Let us conclude this section with a vaguely stated problem.

Problem 7.10. Are there further nonartificial applications of Theorem 4.1? In particular, is there any application which needs the full strength of the theorem?

Although I have searched unsuccessfully for such an application, I still believe that Theorem 4.1 can be exploited further. Many natural classes of sequences, such as the set of all sequences that generate a subspace isomorphic to an $\ell_{p}$ space, concern hereditary properties of subspaces. However, Theorem 4.1 has nothing interesting to say about such properties, since it is trivial that if $\sigma$ is a hereditary property of subspaces, then there is a subspace such that either all its subspaces are in $\sigma$ or none of them are. This indicates that any application of Theorem 4.1 is likely to involve a "clever" choice of sequences, which gives me hope that there are interesting applications that have not yet been found. As for using the full strength of the theorem, this could mean applying it to a set of sequences which is analytic but not Borel (see [Bos] for some good examples of natural Banach-space properties which are genuinely analytic), but it would also be good to see an application which involved a strategy for $\mathrm{S}$ that was more complicated than simply alternating between two subspaces.

\section{Appendix}

In this section we indicate briefly directions in which the results of Sections 2 to 5 cannot be strengthened. The most obvious thing to show is that not every set is weakly Ramsey. That follows from the following statement, that there need not be a subspace in which the game is even approximately determined. The proof, of course, uses the axiom of choice, and in fact the continuum hypothesis as well, although this is not so obviously necessary. (In fact, in [BLA1] it is shown that Martin's axiom suffices.) It would be interesting to know whether the axiom of determinacy implied that every set was weakly Ramsey.

In order to apply the continuum hypothesis, it is essential that the number of strategies for both players of the game is at most $2^{\aleph_{0}}$. However, this is not true with the definition of the game given earlier, since both players have $2^{\aleph_{0}}$ 
possible moves at each stage. Fortunately, this problem is not too serious. First of all, one can restrict each of P's moves to a countable dense set. (For example, insist that they are rational linear combinations of the block basis presented by S.) To deal with S, notice that it is not important for the game that $\mathrm{S}$ should declare an entire block subspace all at once - it is enough if $\mathrm{S}$ presents the block basis one vector at a time, letting $\mathrm{P}$ choose a linear combination of blocks at some finite stage. If in addition $\mathrm{S}$ is restricted to a countable dense set, then the number of strategies for both players is $2^{\aleph_{0}}$ as required. I am grateful to Joan Bagaria and Jordi Lopez Abad for pointing out the necessity of this remark.

TheOREM 8.1. Let $\Delta$ be the constant sequence $(1 / 2,1 / 2, \ldots)$. For every $X$ there exists a set $\sigma \subset \Sigma(X)$ such that in no subspace $Y \subset X$ does $P$ have a winning strategy for the game $\sigma_{\Delta}[Y]$ and in no subspace $Y \subset X$ does $S$ have a winning strategy for the game $\sigma^{\mathrm{c}}[Y]$.

Proof. Well order the set of all possible pairs consisting of a pair $(Y, \phi)$, where $Y$ is a block subspace of $X$ and $\phi$ is either a strategy for $\mathrm{P}$ or a strategy for $\mathrm{S}$ inside $Y$. Do this so that each pair has countably many predecessors. We inductively define for each ordinal $\alpha$ a sequence $s_{\alpha}$ as follows. Suppose the $\alpha^{\text {th }}$ pair in the well ordering is the pair $\left(Y_{\alpha}, \phi_{\alpha}\right)$ and that $\phi_{\alpha}$ is a strategy for $\mathrm{P}$ in $Y_{\alpha}$. Since there are only countably many sequences $s_{\beta}$ with $\beta<\alpha$, $\mathrm{S}$ can easily find a sequence of moves such that the sequence chosen by $\mathrm{P}$ is not within $\Delta$ of any of these $s_{\beta}$. In that case, let $s_{\alpha}$ be the sequence resulting from such a play from $\mathrm{S}$ (with $\mathrm{P}$ playing the strategy $\phi_{\alpha}$ ). Call such an $s_{\alpha}$ an S-sequence. Similarly, if $\phi_{\alpha}$ is a strategy for S, then $\mathrm{P}$ can easily choose a sequence $s_{\alpha}$ against this strategy not within $\Delta$ of any $s_{\beta}$ with $\beta<\alpha$. Call this sort of $s_{\alpha}$ a P-sequence. Note that, in particular, we have guaranteed that no $\mathrm{S}$-sequence is within $\Delta$ of any $\mathrm{P}$-sequence.

Now let $\sigma$ be the set of all $\mathrm{P}$-sequences. Given any strategy for $\mathrm{S}$ and any subspace $Y, \mathrm{P}$ can play against this strategy and produce a $\mathrm{P}$-sequence. Thus, $\mathrm{S}$ has no strategy for any $\sigma^{\mathrm{c}}[Y]$. Similarly, given any strategy for $\mathrm{P}$ and any subspace $Y, \mathrm{~S}$ can force $\mathrm{P}$ to produce an $\mathrm{S}$-sequence, which is not in $\sigma_{\Delta}$. So $\mathrm{P}$ does not have a winning strategy for $\sigma_{\Delta}[Y]$.

Recall the definition of an asymptotic set given earlier. A fundamental fact in Banach space theory is that there exist spaces $X$ such that the unit sphere $S(X)$ contains two asymptotic sets $A$ and $B$ and $\delta>0$ with $\|x-y\| \geqslant \delta$ for every $x \in A$ and $y \in B$. It follows from work of Milman [Mi] that such sets exist in Tsirelson's space. A major breakthrough due to Odell and Schlumprecht [OS] was the discovery of such sets in $\ell_{p}$ for $1 \leqslant p<\infty$. The results of [Mi], [OS] and [G5] in fact show that a space has this property if and only if it does not contain $c_{0}$ [OS, Corollary 1]. 
Corollary 8.2. If $X$ does not contain $c_{0}$, then there exist subsets $\sigma$ and $\tau$ of $\Sigma(X)$, and $\Gamma>0$, such that $P$ has a winning strategy for every $\sigma[Y]$ and every $\tau[Y]$ but $P$ has no winning strategy for any $\left(\sigma_{\Gamma} \cap \tau_{\Gamma}\right)[Y]$ and $S$ has no winning strategy for any $(\sigma \cap \tau)^{\mathrm{c}}[Y]$.

Proof. Let $\mathcal{A}$ and $\mathcal{B}$ be asymptotic sets such that $\|x-y\| \geqslant \delta$ for every $x \in \mathcal{A}$ and $y \in \mathcal{B}$. Let $\rho$ be a set given by Theorem 8.1. Let $\sigma$ be the set of all sequences $\left(x_{n}\right)_{n=1}^{\infty}$ either in $\rho$ or such that $x_{1} \in \mathcal{A}$ and let $\tau$ be the same with $\mathcal{B}$. Clearly $\mathrm{P}$ has winning strategies in every subspace $Y$ for $\sigma[Y]$ and for $\tau[Y]$. Let $\Gamma$ be a sequence with $\gamma_{1}=\min (\delta / 2,1 / 3)$. Then $\sigma_{\Gamma} \cap \tau_{\Gamma}=\rho_{\Gamma} \subset \rho_{\Delta}$ and $\sigma \cap \tau=\rho$, so the result follows from the property of $\rho$.

If $\sigma$ and $\tau$ are given by this corollary, then $\sigma_{\Delta / 2}$ and $\tau_{\Delta / 2}$ are obviously completely weakly Ramsey, but their intersection is not even in an approximate sense completely weakly Ramsey.

LEMma 8.3. If $X$ does not contain $c_{0}$ then there exists a large subset $\sigma \subset \Sigma(X)$ and $\Gamma>0$ such that $\sigma_{\Gamma}$ is $\mathrm{D}$-*-nowhere dense.

Proof. Let $\mathcal{A}$ and $\Gamma$ be as in the proof of the previous corollary. Let $\sigma$ be the set of sequences $\left(x_{n}\right)_{n=1}^{\infty}$ with $x_{n} \in \mathcal{A}$ for every $n$. Clearly $\sigma$ is large. However, given any basic open set $[A ; Z]$, pick $z \in Z \cap \mathcal{B}$. Then $[(A, z) ; Z] \cap \sigma_{\Gamma}$ $=\emptyset$.

\section{Recent developments}

As mentioned in the introduction, this paper is the final version of a preprint [G3] that has been around for several years. I can offer no excuse for this state of affairs, but am glad to remedy it now. I am grateful to certain people for drawing my attention to mistakes in the preprint. Valentin Ferenczi pointed out that the proof of Theorem 8 in that paper (the statement that Dopen sets are weakly Ramsey) was incorrect. (On the other hand, the proof in [G2] was correct.) This was later pointed out to me again by Joan Bagaria and Jordi Lopez Abad, who also said that they found the proof of the main theorem (corresponding to Theorem 4.1 in this paper) hard to follow. In response, I have made my presentation clearer in many respects. I have also noticed and corrected a mistake in the proof of Lemma 9, which corresponds to Lemma 4.12 here. (Lemma 4.11 does not have a counterpart in the preprint, and was the missing step.)

Recently Bagaria and Lopez Abad have given alternative proofs of the main results of this paper, and have extended some of them [BLA1,2]. Their

arguments are along similar lines to the ones here, but are expressed in a 
language more familiar to logicians. For example, they speak of dense generic filters when carrying out their diagonalizations. Under additional set-theoretic hypotheses they extend Theorem 4.1 to $\Sigma_{2}^{1}$-sets, that is, continuous images of coanalytic sets. Their work was carried out before I revised my preprint.

DPMms, University of Cambridge, Wilberforce Road, Cambridge CB3 OWB, UK

E-mail address: wtg10@dpmms.cam.ac.uk

\section{REFERENCES}

[AD] S. A. Argyros and I. Deliyanni, Examples of asymptotic $\ell_{1}$ Banach spaces, Trans. Amer. Math. Soc. 349 (1997), 973-995.

[BLA1] J. BAGARia and J. López-Abad, Weakly Ramsey sets in Banach spaces, Adv. Math. 160 (2001), 133-174.

[BLA2] Determinacy and weakly Ramsey sets in Banach spaces, Trans. Amer. Math. Soc. 354 (2002), 1327-1349.

[B] S. Banach, Théorie des Opérations Linéaires, Éditions Jacques Gabay, Sceaux, 1993 (Reprint of 1932 original).

[Bo] B. Bollobás, Combinatorics. Set Systems, Hypergraphs, Families of Vectors and Combinatorial Probability, Cambridge University Press, Cambridge, 1986.

[Bos] B. Bossard, Théorie descriptive des ensembles en géometrie des espaces de Banach, Ph.D. Thesis, Université de Paris VI (1994).

[CS] T. J. Carlson and S. G. Simpson, A dual form of Ramsey's theorem, Adv. in Math. 53 (1984), 265-290.

[C] P. G. Casazza, handwritten notes.

[CO] P. G. Casazza and E. Odell, Tsirelson's space and minimal subspaces, Longhorn Notes, University of Texas, Texas Functional Analysis Seminar (1982-3), 61-72.

[E] E. Ellentuck, A new proof that analytic sets are Ramsey, J. Symbolic Logic 39 (1974), 163-165.

[F1] V. Ferenczi, A uniformly convex hereditarily indecomposable Banach space, Israel J. Math. 102 (1997), 199-225.

[F2] Operators on subspaces of hereditarily indecomposable Banach spaces, Bull. London Math. Soc. 29 (1997), 338-344.

[GP] F. Galvin and K. Prikry, Borel sets and Ramsey's theorem, J. Symbolic Logic 38 (1973), 193-198.

[G1] W. T. Gowers, A new dichotomy for Banach spaces, Geom. Funct. Anal. 6 (1996), 1083-1093.

[G2] - A new dichotomy for Banach spaces, preprint.

[G3] - Analytic sets and games in Banach spaces, preprint IHES M/94/42.

[G4] A solution to Banach's hyperplane problem, Bull. London Math. Soc. 26 (1994), 523-530.

[G5] Lipschitz functions on classical spaces, Europ. J. Combin. 13 (1992), 141151.

[G6] A Banach space not containing $c_{0}, \ell_{1}$ or a reflexive subspace, Trans. Amer. Math. Soc. 344 (1994), 407-420.

[GM1] W. T. Gowers and B. Maurey, The unconditional basic sequence problem, J. Amer. Math. Soc. 6 (1993), 851-874.

[GM2] Banach spaces with small spaces of operators, Math. Ann. 307 (1997), 543-568.

[H] P. Habala, A Banach space all of whose subspaces fail the Gordon-Lewis property, Math. Ann. 310 (1998), 197-219. 
[J] T. Jech, Set Theory, Academic Press, New York (1978).

$[\mathrm{K}$ T-J] R. A. Komorowski and N. Tomczak-Jaegermann, Banach spaces without local unconditional structure, Israel J. Math. 89 (1995), 205-226.

[LT] J. Lindenstrauss and L. Tzafriri, Classical Banach Spaces. I. Sequence Spaces, Springer-Verlag, New York, 1977.

[M] A. R. D. Mathias, Happy families, Ann. Math. Logic 12 (1977), 59-111.

[Mi] V. D. Milman, Spectrum of bounded continuous functions which are given on the unit sphere of a Banach space, Funct. Anal. Appl. 3 (1969), 67-79 (translated from Russian).

[N-W] C. St. J. A. Nash-Williams, On well-quasi-ordering transfinite sequences, Proc. Cambridge Philos. Soc. 61 (1965), 33-39.

[OS] E. Odell and T. Schlumprecht, The distortion problem, Acta Math. 173 (1994), 259-281.

[S] J. Silver, Every analytic set is Ramsey, J. Symbolic Logic 35 (1970), 60-64.

[Sz] A. Szankowski, Subspaces without the approximation property, Israel J. Math. 30 (1978), 123-129.

(Received March 30, 2000) 